\title{
Structures magnétiques et symétries cristallines
}

\section{J. Schweizer}

CEA-Grenoble, DRFMC/SPSMS/MDN, 38054 Grenoble cedex, France

\section{INTRODUCTION}

Nous nous intéressons aux moments magnétiques dans la matière cristallisée. Ils sont portés par des atomes particuliers comme les métaux de transition, les atomes de terres rares ou les atomes d'actinides. Ces moments magnétiques sont soumis à des interactions d'échange et l'énergie libre d'interaction magnétique peut s'écrire:

$$
\mathrm{U}=-\sum_{\mathrm{i}, \mathrm{j}} \mathrm{J}_{\mathrm{ij}} \overrightarrow{\mathrm{m}}_{\mathrm{i}} \overrightarrow{\mathrm{m}}_{\mathrm{j}}
$$

A haute température, dans l'état paramagnétique, ces moments sont désordonnés, c'est à dire qu'il n'y a pas d'ordre à longue distance. Mais il existe, à courte distance, une certaine tendance à l'ordre: ce sont des fluctuations magnétiques. Lorsqu'on refroidit le cristal, la portée de ces fluctuations augmente et, à un moment donné, ces fluctuations se transforment en un ordre à grande distance : une structure magnétique s'est établie.

Nous allons analyser ces structures magnétiques en utilisant la théorie de Landau.

Au dessus de la température d'ordre, on peut classer les fluctuations dans l'état paramagnétique selon des modes compatibles avec les symétries du cristal. Dans ces symétries, il faudra distinguer entre

- les symétries de translation (propres au réseau)

- les symétries de rotation-inversion (propres au motif)

Au moment de la mise en ordre (mise en ordre du 2eme ordre pour pouvoir appliquer la théorie de Landau), un de ces modes s'établit de façon stable, tandis que les autres modes s'évanouissent.

En utilisant les concepts de la théorie des groupes, nous verrons que chaque mode correspond à une représentation irréductible du groupe des symétries. 


\section{ON UTILISE LES PROPRIÉTÉS DE TRANSLATION DU RÉSEAU}

On commence par partir d'un cas simplifié où il n'y a qu'un seul atome magnétique par maille (on le supposera à l'origine de la maille) et où les interactions sont de type Heisenberg.

L'énergie libre s'écrit alors:

$$
\mathrm{U}=-\sum_{1, \mathrm{r}} \mathrm{J}_{\mathrm{n}} \cdot \overrightarrow{\mathrm{m}}_{1} \overrightarrow{\mathrm{m}}_{\mathrm{r}}
$$

où 1 et l' repèrent l'origine de chaque maille dans le cristal.

Parce que les moments $\vec{m}_{1}$ sont aux noeuds d'un réseau, on démontre (voir annexe) que, quelle que soit la distribution des moments dans le cristal, on peut écrire:

$$
\overrightarrow{\mathrm{m}}_{1}=\int_{\substack{\text { lere zone } \\ \text { Brillowin }}} \mathrm{d}^{3} \mathrm{k} \overrightarrow{\mathrm{m}}(\overrightarrow{\mathrm{k}}) \mathrm{e}^{-\overrightarrow{\mathrm{k}} \mathrm{k}} \mathrm{i}
$$

où $\overrightarrow{\mathrm{m}}(\overrightarrow{\mathrm{k}})$ est une distribution vectorielle continue et périodique dans le réseau réciproque.

L'énergie libre des interactions de Heisenberg s'écrit alors :

$$
U=-\sum_{i, t^{\prime}} J_{1 I^{\prime}} \iint d^{3} k d^{3} k^{\prime} \vec{m}(\vec{k}) \vec{m}\left(\vec{k}^{*}\right) e^{-\vec{k} \vec{t}} e^{-i \vec{k}^{\prime} \vec{r}^{*}}
$$

On introduit alors:

$$
e^{i \vec{k} \bar{T}^{\prime}} e^{-i \vec{k} \vec{k}}=1
$$

et on obtient :

$$
U=-\sum_{1, l^{\prime}} \iint d^{3} k d^{3} k^{k} \vec{m}(\vec{k}) \vec{m}\left(\overrightarrow{k^{\prime}}\right) e^{-i \vec{j}^{\prime}\left(\vec{k}+\vec{k}^{\prime}\right)} J_{1 !} e^{-i \vec{k}\left(\vec{i}-\overrightarrow{i^{\prime}}\right)}
$$

On remarque que la somme sur 1 :

$$
\sum_{i} J_{11} e^{-i \vec{k}(\vec{i}-\vec{H})}=J(\vec{k})
$$

est la transformée de Fourier des interactions magnétiques et qu'elle est indépendante de l', c'est à dire du noeud du réseau à partir duquel on fait cette transformée de Fourier, ce qui nous donne pour l'énergie libre:

$$
U=-\iint d^{3} k d^{3} k^{*} \vec{m}(\vec{k}) \vec{m}\left(\overrightarrow{k^{\prime}}\right) J(\vec{k}) \cdot \sum_{r} e^{-i \vec{i}\left(\vec{k}^{\prime}+\vec{k}^{\prime}\right)}
$$

Or, la somme sur un réseau, $\sum_{\vec{r}} \mathrm{e}^{-\overrightarrow{\mathrm{i}}(\overrightarrow{\mathrm{k}}+\overrightarrow{\mathrm{k}})}$ est nulle, sauf si le vecteur $\overrightarrow{\mathrm{k}}+\overrightarrow{\mathrm{k}}$ aboutit à un noeud du réseau réciproque $\vec{\tau}$; 


$$
\sum_{\eta^{\prime}} \mathrm{e}^{\left.-\mathrm{j}^{\prime} \overrightarrow{\mathrm{k}}^{\prime}+\overrightarrow{\mathrm{k}}^{\prime}\right)}=\mathrm{N} \delta\left(\overrightarrow{\mathrm{k}}+\overrightarrow{\mathrm{k}}^{\mathrm{k}}-\vec{\tau}\right)
$$

où $\mathrm{N}$ correspond au nombre de mailles dans le cristal. En particulier, dans la première zone de Brillouin, $\delta\left(\vec{k}+\vec{k}^{\prime}-\vec{\tau}\right)$ n'est différent de zero que pour $\vec{k}=-\vec{k}$. L'énergie libre s'écrit alors comme une intégrale simple sur $\overrightarrow{\mathrm{k}}$ :

$$
U=-N \int d^{3} k \vec{m}(\vec{k}) \vec{m}(-\vec{k}) J(\vec{k})
$$

Jusque là, nous n'avons fait que des mathématiques sur réseaux, en fait sur des vecteurs (les moments magnétiques) qui sont situés aux noeuds d'un réseau. Faisons maintenant un peu de physique.

La fonction $J(\vec{k})$ est maximum pour une valeur particulière $\vec{k}_{0}$ du vecteur $\vec{k}$. C'est le vecteur de propagation qui minimise l'expression de l'énergie libre donnée par (9) et que choisit la structure magnétique au moment où elle s'établit. L'énergie libre ne s'écrit plus alors comme une intégrale sur $\overrightarrow{\mathrm{k}}$ mais comme un simple produit :

$$
U=-\mathrm{N} J\left(\overrightarrow{\mathrm{k}}_{0}\right) \overrightarrow{\mathrm{m}}\left(\overrightarrow{\mathrm{k}}_{0}\right) \cdot \overrightarrow{\mathrm{m}}\left(-\overrightarrow{\mathrm{k}}_{0}\right)
$$

Dans la suite, pour simplifier les notations, nous utiliserons $\overrightarrow{\mathrm{k}}$ au lieu de $\overrightarrow{\mathrm{k}}_{0}$ et $\overrightarrow{\mathrm{m}}^{\overrightarrow{\mathrm{k}}}$ au lieu de $\overrightarrow{\mathrm{m}}\left(\overrightarrow{\mathrm{k}}_{0}\right)$.

La décomposition (3), valable pour n'importe quelle distribution de moments, se limite alors à :

$$
\overrightarrow{\mathrm{m}}_{1}=\overrightarrow{\mathrm{m}}^{\overrightarrow{\mathrm{k}}} \mathrm{e}^{-\mathrm{i} \overrightarrow{\mathrm{k}} \hat{\mathrm{i}}}
$$

qui n'est rien d'autre qu'une fonction de Bloch.

Cependant, pour s'assurer que les moments $\overrightarrow{\mathrm{m}}_{1}$, sur chaque noeud du réseau, sont des vecteurs réels, on associe, si c'est nécessaire, dans le passage de (3) à (11), le vecteur de propagation $-\vec{k}$ au vecteur de propagation $\overrightarrow{\mathrm{k}}$ :

$$
\overrightarrow{\mathrm{m}}_{\mathrm{j}}=\overrightarrow{\mathrm{m}}^{\overrightarrow{\mathrm{k}}} \mathrm{e}^{-i \overrightarrow{\mathrm{k}} \hat{T}}+\overrightarrow{\mathrm{m}}^{-\vec{k}} \mathrm{e}^{i \overrightarrow{\mathrm{k}} \hat{l}}
$$

avec, comme condition pour que cette somme soit réelle :

$$
\overrightarrow{\mathrm{m}}^{-\overrightarrow{\mathrm{k}}}=\left(\overrightarrow{\mathrm{m}}^{\overline{\mathrm{k}}}\right)^{*}
$$

Dans ces formules, $\overrightarrow{\mathrm{m}}^{\overrightarrow{\mathrm{k}}}$ et $\overrightarrow{\mathrm{m}}^{\vec{k}}$ sont appelées les composantes de Fourier de la structure magnétique.

\section{GÉNÉRALISATIONS}

Si, au lieu d'un seul atome magnétique par maille, il y a plusieurs atomes magnétiques dans chaque maille (on dit parfois qu'il y a alors plusieurs réseaux de Bravais magnétiques), les coordonnées de chaque atome magnétique sont données par 


$$
\vec{R}_{\mathrm{lj}}=\overrightarrow{\mathrm{l}}+\overrightarrow{\mathrm{r}}_{\mathrm{j}}
$$

où $\vec{i}$ représente les coordonnées de l'origine de la maille et $\vec{r}_{\mathrm{j}}$ celles de l'atome magnétique $\mathrm{j}$ dans la maille. Au lieu des développements (11) ou (12), on développe les moments magnétiques en utilisant autant de composantes de Fourier qu'il y a d'atomes magnétiques dans la maille :

$$
\begin{aligned}
& \overrightarrow{\mathrm{m}}_{\mathrm{j}}=\overrightarrow{\mathrm{m}}_{\mathrm{j}}^{\overrightarrow{\mathrm{k}}} \mathrm{e}^{-\mathrm{i} \overrightarrow{\mathrm{k}} \overrightarrow{\mathrm{j}}} \\
& \overrightarrow{\mathrm{m}}_{\mathrm{jj}}=\overrightarrow{\mathrm{m}}_{\mathrm{j}}^{\overrightarrow{\mathrm{k}}} \mathrm{e}^{-\overrightarrow{\mathrm{k}} \overrightarrow{\mathrm{j}}}+\overrightarrow{\mathrm{m}}_{\mathrm{j}}^{-\overrightarrow{\mathrm{k}}} \mathrm{e}^{\overrightarrow{\mathrm{k}} \hat{\mathrm{i}}}
\end{aligned}
$$

Il faut noter que si les composantes de Fourier $\overrightarrow{\mathrm{m}}_{\mathrm{j}}^{\overrightarrow{\mathrm{k}}}$ dépendent bien de l'atome magnétique $\mathrm{j}$, l'exponentielle (ou les exponentielles) ne porte toujours que sur $\vec{i}$, l'origine de la maille.

L'autre généralisation concerne l'énergie libre. Au lieu d'interactions isotropes de Heisenberg, on considère une énergie libre très générale, où les composantes $\alpha$ et $\beta$ des moments magnétiques se couplent à d'ordre 2. Au lieu de partir de l'expression (2), on part de

$$
\mathrm{U}=-\sum_{\mathrm{i}, \mathbb{f}^{\prime}} \sum_{\mathrm{j}, j} \sum_{\alpha, \beta} \mathrm{J}_{\mathrm{II} \mathrm{j}^{\prime} \mathrm{j}^{\prime} \alpha \beta} \mathrm{m}_{\mathrm{lj}, \alpha} \mathrm{m}_{\mathrm{r}^{\prime} \mathrm{j}^{\prime} \beta}
$$

et, au lieu d'arriver à l'expression (10), on arrive à :

$$
\mathrm{U}=-\mathrm{N} \sum_{\mathrm{j}, \mathrm{j}^{t}} \sum_{\alpha, \beta} \mathrm{J}_{\mathrm{j}, \alpha \beta}(\overrightarrow{\mathrm{k}}) \mathrm{m}_{\mathrm{j} \alpha}^{\overrightarrow{\mathrm{k}}} \mathrm{m}_{\mathrm{j}^{\prime} \beta}^{-\overrightarrow{\mathrm{k}}}
$$

La structure magnétique qui s'établit à la température d'ordre sera celle qui minimise l'énergie libre donnée par (18), en choisissant le vecteur de propagation $\overrightarrow{\mathrm{k}}$ et aussi en déterminant l'arrangement relatif des composantes $m_{j \alpha}^{\bar{k}}$.

\section{EXEMPLES DE STRUCTURES MAGNÉTIQUES}

Nous classerons les structures magnétiques selon la nature de leur vecteur de propagation.

\subsection{Vecteur de propagation nul : $\overrightarrow{\mathrm{k}}=(0,0,0)$}

Tout arrangement de la première maille est reproduit intégralement dans les autres mailles.

C'est là qu'on trouve les structures ferromagnétiques où tous les moments sont égaux et parallèles. 


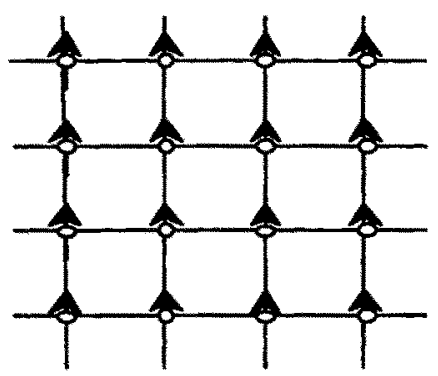

Figure 1: Structure ferromagnêtique $\overrightarrow{\mathrm{k}}=(0,0,0)$

Mais c'est là qu'on peut trouver des structures antiferromagnétiques colinéaires ou triangulaires où, à l'intérieur de chaque maille, les moments d'atomes identiques, mais non reliés entre eux par une translation du réseau, sont opposés ou forment un triangle.

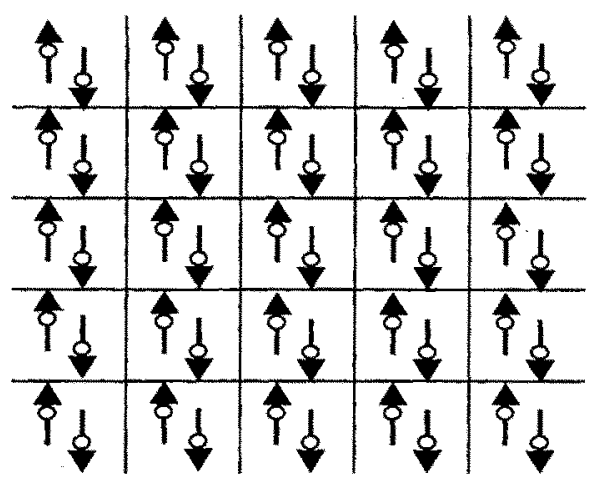

Figure 2: Structure antiferromagnếtique

$$
\overrightarrow{\mathrm{k}}=(0,0,0)
$$

\subsection{Vecteur de propagation fractionnaire}

Par exemple $\overrightarrow{\mathrm{k}}=(1 / 2,0,0)$ qui, par application de $(15)$ entraîne $\overrightarrow{\mathrm{m}}_{\mathrm{j} j}=\overrightarrow{\mathrm{m}}_{\mathrm{j}} \mathrm{e}^{-\mathrm{i} \pi \mathrm{n}}$ où $\mathrm{n}$ est un nombre entier. Il s'agit d'une structure antiferromagnétique, où les moments restent égaux mais changent de signe d'une maille à l'autre avec une propagation selon $x$. La maille magnétique est ici deux fois plus grande que la maille chimique. 


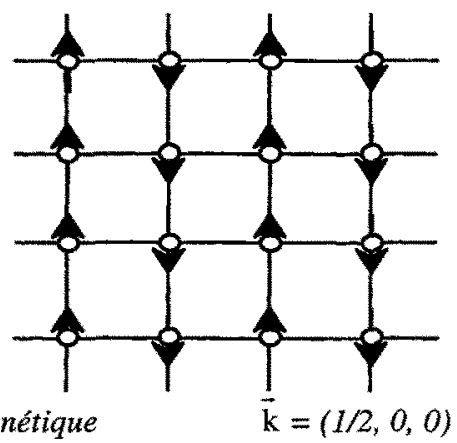

Figure 3: Structure antiferromagnétique

$$
\overrightarrow{\mathrm{k}}=(1 / 2,0,0)
$$

\subsection{Vecteur de propagation incommensurable}

Par exemple $\vec{k}=\left(k_{x}, 0,0\right)$ qui, par application de (16) entraîne :

$$
\overrightarrow{\mathrm{m}}_{\mathrm{ij}}=\overrightarrow{\mathrm{m}}_{\mathrm{j}}^{\overrightarrow{\mathrm{k}}} \mathrm{e}^{-\mathrm{i} \overrightarrow{\mathrm{k}} \mathbf{I}}+\overrightarrow{\mathrm{m}}_{\mathrm{j}}^{-\overrightarrow{\mathrm{k}}} \mathrm{e}^{\overrightarrow{\mathrm{k}} \overline{\mathrm{I}}}
$$

Si $\overrightarrow{\mathrm{m}}_{\mathrm{j}}^{\overrightarrow{\mathrm{k}}}$ est un vecteur réel (à une dimension), $\overrightarrow{\mathrm{m}}_{\mathrm{j}}^{\overrightarrow{\mathrm{k}}}=\overrightarrow{\mathrm{m}}_{j}^{-\overrightarrow{\mathrm{k}}}=\overrightarrow{\mathrm{u}}_{\mathrm{j}}$ et on a:

$$
\overrightarrow{\mathrm{m}}_{\mathrm{jj}}=2 \overrightarrow{\mathrm{u}}_{\mathrm{i}} \cos \overrightarrow{\mathrm{k}} \overrightarrow{\mathrm{i}}
$$

Il s'agit d'une structure sinusoïdale ou modulée se propageant selon $\mathrm{x}$.

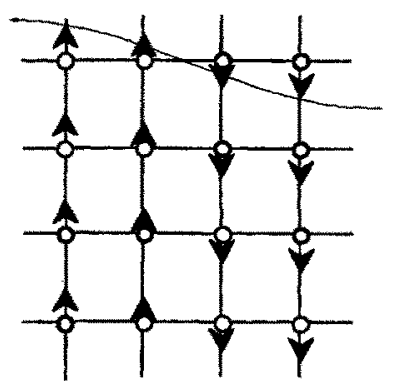

Figure 4 : Structure modulée

$$
\overrightarrow{\mathrm{k}}=\left(k_{x}, 0,0\right)
$$

Si $\overrightarrow{\mathrm{m}}_{j}^{\overrightarrow{\mathrm{k}}}$ est un vecteur complexe (à deux dimensions), $\overrightarrow{\mathrm{m}}_{\mathrm{j}}^{\overrightarrow{\mathrm{k}}}=\overrightarrow{\mathrm{u}}_{\mathrm{j}}+\overrightarrow{i \mathrm{v}} \overrightarrow{\mathrm{j}}_{\mathrm{j}}$. On a alors par application de (13) $\overrightarrow{\mathrm{m}}_{j}^{-\vec{k}}=\vec{u}_{j}-i \vec{v}_{j}$, ce qui entraîne:

$$
\overrightarrow{\mathrm{m}}_{\mathrm{ij}}=2\left(\mathrm{u}_{\mathrm{j}} \cos \overrightarrow{\mathrm{k}} \overrightarrow{\mathrm{l}}+\overrightarrow{\mathrm{v}}_{\mathrm{i}} \sin \overrightarrow{\mathrm{k}} \mathrm{l}\right)
$$


Il s'agit cette fois d'une structure hélicoïdale, se propageant toujours selon $\mathrm{x}$. Cette hélice est a priori elliptique. Elle sera circulaire si les deux vecteurs $\overrightarrow{\mathrm{u}}$ et $\overrightarrow{\mathrm{v}}$ sont orthogonaux et égaux.

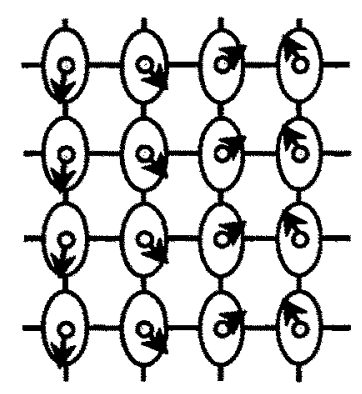

Figure 5 : Structure hélicoïdale

$$
\overrightarrow{\mathbf{k}}=\left(k_{x}, 0,0\right)
$$

\section{SECTIONS EFFICACES COHÉRENTES AUX NEUTRONS}

Pour la diffusion nucléaire, si on néglige la partie dépendant du spin, chaque noyau diffuse les neutrons avec une amplitude :

$$
a_{N}=b_{j}
$$

amplitude indépendante de $\mathrm{Q}$, égale à la longueur de Fermi caractéristique de chaque noyau et que lon trouve dans des tables.

Ce qui fait pour la section efficace cohérente, après avoir sommé les amplitudes sur tous les noyaux et pris le carré du module de cette somme :

$$
\left(\frac{\mathrm{d} \sigma}{\mathrm{d} \Omega}\right)_{\mathrm{N}}=\left|\sum_{\mathrm{l}, \mathrm{j}} \mathrm{b}_{\mathrm{j}} \mathrm{e}^{+\mathrm{i} \ddot{\mathrm{Q}} \tilde{\mathrm{R}}_{i i}}\right|^{2}=\mathrm{N}^{2} \sum_{\vec{\tau}}\left|\mathrm{F}_{\mathrm{N}}\right|^{2} \delta(\overrightarrow{\mathrm{Q}}-\vec{\tau})
$$

Il n'y a de la section efficace cohérente que sur les noeuds $\vec{\tau}$ du réseau réciproque, et l'intensité pour chacune de ces réflexions de Bragg correspond au carré du module du facteur de structure nucléaire $\mathrm{F}_{\mathrm{N}}$, avec, pour ce facteur de structure une somme, à l'intérieur de la maille élémentaire :

$$
F_{N}=\sum_{j} b_{j} e^{+i Q \tilde{F}_{j}} e^{-w_{j}}
$$

Pour la diffusion magnétique, chaque moment magnétique diffuse les neutrons avec une amplitude :

$$
\mathrm{a}_{\mathrm{M}}(\overrightarrow{\mathrm{Q}})=\mathrm{p} \vec{\sigma} \overrightarrow{\mathrm{m}}_{\perp} \mathrm{f}(\overrightarrow{\mathrm{Q}})
$$

- où p est une longueur caractéristique qui vaut $0.269610^{-12} \mathrm{~cm}$,

- $\vec{\sigma}$ est l'opérateur unitaire caractéristique du spin du neutron et représenté par les matrices de Pauli, - le moment magnétique $\overrightarrow{\mathrm{m}}$ est exprimé en magnétons de Bohr $\left(\mu_{\mathrm{B}}\right)$ et $\overrightarrow{\mathrm{m}}_{1}$ est sa projection sur le plan perpendiculaire au vecteur de diffusion $\overrightarrow{\mathrm{Q}}$ 
- le facteur de forme $f(\vec{Q})$ représente la dépendance angulaire de la diffusion, due à la taille non négligeable du nuage électronique magnétique. Les facteurs de forme magnétiques, eux aussi, sont tabulés pour les différents ions magnétiques.

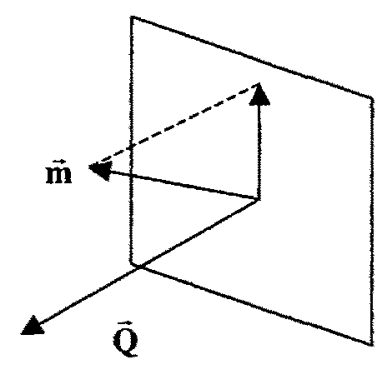

Figure 6: Projection $\overrightarrow{\mathrm{m}}_{\perp}$ du vectear $\overrightarrow{\mathrm{m}}$

Pour la section efficace magnétique cohérente, on somme les amplitudes et on prend le carré du module de la même façon que pour la section efficace nucléaire :

$$
\left(\frac{\mathrm{d} \sigma}{\mathrm{d} \Omega}\right)_{M}=\left|\sum_{\mathrm{h}_{i j}} \mathrm{a}_{\mathrm{Mij}}(\overrightarrow{\mathrm{Q}}) \mathrm{e}^{+\mathrm{i} \overrightarrow{\mathrm{Q}} \overrightarrow{\mathrm{R}}_{i j}}\right|^{2}
$$

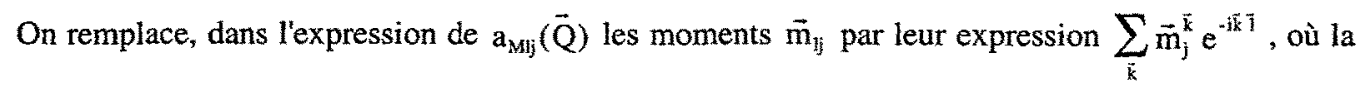
somme sur $\overrightarrow{\mathrm{k}}$ porte sur les deux vecteurs $\overrightarrow{\mathrm{k}}$ et $-\overrightarrow{\mathrm{k}}$. On arrive ainsi ̀̀ :

$$
\left(\frac{\mathrm{d} \sigma}{\mathrm{d} \Omega}\right)_{\mathrm{M}}=\mathrm{N}^{2} \sum_{\overrightarrow{\mathrm{k}}} \sum_{\vec{\tau}}\left|\overrightarrow{\mathrm{F}}_{\mathrm{M} \perp}\right|^{2} \delta(\overrightarrow{\mathrm{Q}}-\overrightarrow{\mathrm{k}}-\vec{\tau})
$$

Il n'y aura dintensité magnétique que pour les deux positions $\vec{Q}=\vec{\tau}+\vec{k}$ et $\vec{Q}=\vec{\tau}-\vec{k}$ du vecteur de diffusion de part et d'autre des nœuds du réseau réciproque.

Il faut noter aussi que, contrairement au cas nucléaire, le facteur de structure est maintenant un vecteur :

$$
\overrightarrow{\mathrm{F}}_{\mathrm{M}}=\mathrm{p} \sum_{j} \overrightarrow{\mathrm{m}}_{j}^{\overrightarrow{\mathrm{k}}} \mathrm{f}_{j}(\overrightarrow{\mathrm{Q}}) \mathrm{e}^{+\mathrm{i} \overrightarrow{\mathrm{Q}} \overrightarrow{\mathrm{i}}_{\mathrm{j}}} \mathrm{e}^{-\mathrm{W}_{j}}
$$

et que seule sa projection $\overrightarrow{\mathrm{F}}_{\mathrm{ML}}$ sur le plan perpendiculaire au vecteur de diffusion $\overrightarrow{\mathrm{Q}}$ intervient dans l'expression de la section efficace.

Nous reprenons les exemples de structures magnétiques présentés au paragraphe précédent. 
Vecteur de propagation nul : $\overrightarrow{\mathrm{k}}=(0,0,0)$

Structures ferromagnétiques, mais parfois aussi antiferromagnétiques ou triangulaires :

$$
\left(\frac{\mathrm{d} \sigma}{\mathrm{d} \Omega}\right)_{\mathrm{M}}=\mathrm{N}^{2} \sum_{\overrightarrow{\mathrm{F}}}\left|\overrightarrow{\mathrm{F}}_{\mathrm{M} L}\right|^{2} \delta(\overrightarrow{\mathrm{Q}}-\vec{\tau})
$$

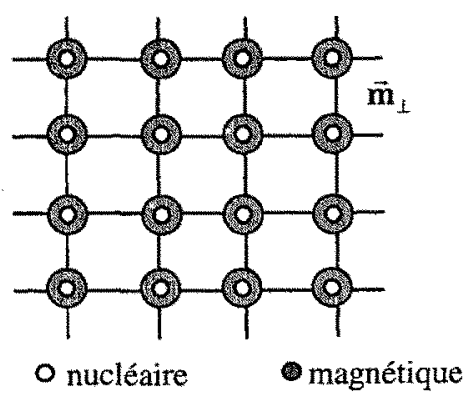

Figure 7 : Réflexions magnétiques pour $\overrightarrow{\mathrm{k}}=(0,0,0)$

Nous nous retrouvons, comme pour la diffusion nucléaire, avec de la section efficace magnétique uniquement sur les noeuds du réseau réciproque. Comme $\overrightarrow{\mathrm{m}}_{j}^{\vec{k}}=\overrightarrow{\mathrm{m}}_{0 j}$, où $\overrightarrow{\mathrm{m}}_{0 j}$ représente le moment de l'atome $\mathrm{j}$ dans la maille initiale du cristal, on a :

$$
\vec{F}_{M}=p \sum_{j} \vec{m}_{j}^{\vec{k}} f_{j}(\vec{Q}) e^{+i \vec{Q} \vec{f}_{j}} e^{-W_{j}}=p \sum_{j} \vec{m}_{0 j} f_{j}(\vec{Q}) e^{+i \vec{Q} \vec{r}_{j}} e^{-W_{j}}
$$

\section{Vecteur de propagation fractionnaire :}

Structures antiferromagnétiques.

$$
\left(\frac{\mathrm{d} \sigma}{\mathrm{d} \Omega}\right)_{M}=\mathrm{N}^{2} \sum_{\bar{z}}\left|\overrightarrow{\mathrm{F}}_{M L}\right|^{2} \delta(\overrightarrow{\mathrm{Q}} \pm \overrightarrow{\mathrm{k}}-\vec{\tau})
$$

Le vecteur de propagation se trouve souvent sur la surface de la zone de Brillouin, ce qui entraîne que les réflexions magnétiques sont généralement à mi-distance entre deux noeuds du réseau réciproque, avec des vecteurs de diffusion $\vec{\tau}+\vec{k}$ et $\vec{\tau}-\vec{k}$ qui se regroupent 2 par 2 pour donner une seule et même réflexion magnétique. 


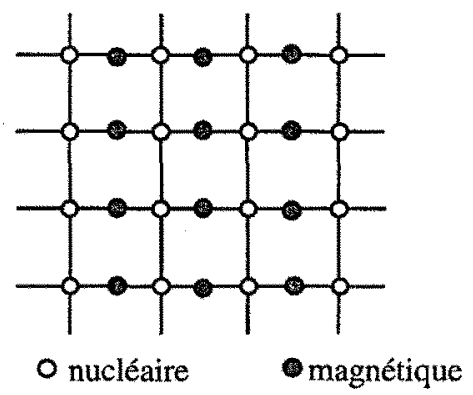

Figure $8:$ Réflexions magnétiques pour $\overrightarrow{\mathrm{k}}=(1 / 2,0,0)$

Comme $\overrightarrow{\mathrm{m}}_{\mathrm{ij}}=\overline{\mathrm{m}}_{\mathrm{j}}^{\overrightarrow{\mathrm{k}}} \mathrm{e}^{-i \pi \mathrm{n}}$ on a $\overrightarrow{\mathrm{m}}_{\mathrm{j}}^{\overrightarrow{\mathbf{k}}}=\overrightarrow{\mathrm{m}}_{0 \mathrm{j}}$ où $\overrightarrow{\mathrm{m}}_{0 \mathrm{j}}$ représente le moment de l'atome $\mathrm{j}$ dans la maille initiale du cristal, et le facteur de structure magnétique s'écrit :

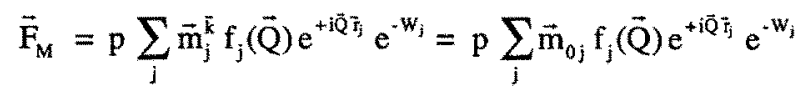

Vecteur de propagation incommensurable: $\overrightarrow{\mathrm{k}}=\left(\mathrm{k}_{\mathrm{x}}, 0,0\right)$

Structures modulées ou hélicoïdales

$$
\left(\frac{d \sigma}{d \Omega}\right)_{M}=\mathrm{N}^{2} \sum_{\vec{\xi}}\left(\left|\overrightarrow{\mathrm{F}}_{\mathrm{M}}\right|^{2} \delta(\overrightarrow{\mathrm{Q}}-\overrightarrow{\mathrm{k}}-\vec{\tau})+\left|\overrightarrow{\mathrm{F}}_{\mathrm{M}}\right|^{2} \delta(\overrightarrow{\mathrm{Q}}+\overrightarrow{\mathrm{k}}-\vec{\tau})\right)
$$

Autour de chaque noeud du réseau réciproque, il y a deux réflexions que l'on appelle les satellites magnétiques:

- une réflexion pour $\overrightarrow{\mathrm{Q}}=\vec{\tau}+\overrightarrow{\mathrm{k}}$, dite satellite $\vec{\tau}^{+}$

- une réflexion pour $\overrightarrow{\mathrm{Q}}=\vec{\tau}-\overrightarrow{\mathrm{k}}$, dite satellite $\vec{\tau}$

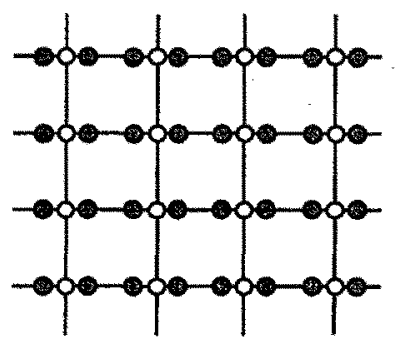

$$
\text { O nucléaire magnétique }
$$

Figure 9: Réflexions magnétiques pour $\overrightarrow{\mathrm{k}}=\left(k_{x}, 0,0\right)$

Pour une structure modulée où $\vec{m}_{\mathrm{lj}}=2 \overrightarrow{\mathrm{u}}_{\mathrm{j}} \cos \overrightarrow{\mathrm{k}} \overrightarrow{\mathrm{l}}$, le facteur de structure magnétique est égal à : 


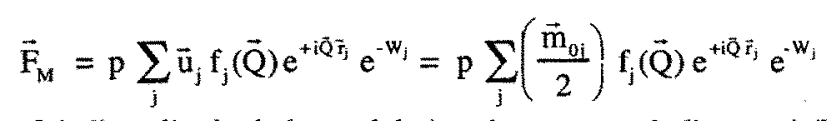

où $\overrightarrow{\mathrm{m}}_{0 \mathrm{j}}$ représente cette fois l'amplitude de la modulation du moment de l'atome $\mathrm{j}$ (le maximum de la sinusoilde).

Pour une structure hélicoïdale, où $\overrightarrow{\mathrm{m}}_{\mathrm{jj}}=2\left(\overrightarrow{\mathrm{u}}_{\mathrm{j}} \cos \overrightarrow{\mathrm{k}} \overrightarrow{\mathrm{l}}+\overrightarrow{\mathrm{v}}_{\mathrm{j}} \sin \overrightarrow{\mathrm{k}} \mathrm{l}\right)$, le facteur de structure magnétique est donné par :

$$
\vec{F}_{M}=p \sum_{j}\left(\vec{u}_{j}+i \vec{v}_{j}\right) f_{j}(\vec{Q}) e^{+i \vec{Q} \vec{t}_{j}} e^{-w w_{j}}
$$

\section{STRUCTURES MAGNÉTIQUES: IDENTIFICATION DU VECTEUR DE PROPAGATION}

La première étape, lorsqu'on cherche à déterminer une structure magnétique, est de faire deux diagrammes de poudre: au dessus et au dessous de la température de mise en ordre. La différence entre les deux diagrammes fait apparâtre les raies magnétiques. Ces raies magnétiques sont reliées aux nœuds du réseau réciproque $\vec{\tau}$ par la relation :

$$
\overrightarrow{\mathrm{Q}}=\vec{\tau} \pm \overrightarrow{\mathrm{k}}
$$

et l'indexation de ces raies magnétiques permet de connattre le vecteur de propagation $\overrightarrow{\mathrm{k}}$.

Il y a 3 méthodes d'indexation des raies magnétiques:

La méthode intuitive qui marche surtout pour des vecteurs $\overrightarrow{\mathrm{k}}$ fractionnaires simples comme $\overrightarrow{\mathrm{k}}=(0,0,0), \overrightarrow{\mathrm{k}}=(1 / 2,0,0)$ ou $\overrightarrow{\mathrm{k}}=(1 / 2,1 / 2,1 / 2)$.

La méthode graphique qui trace sur certains plans bien choisis du réseau réciproque des cercles de rayons égaux aux longueurs des vecteurs $\overline{\mathrm{Q}}$ correspondant aux raies magnétiques. A partir de ces graphiques on cherche, de part et d'autre des nœuds du réseau réciproque, sil existe un vecteur $\vec{k}$ qui satisfait à la relation (35).

La méthode informatique qui liste les longueurs des vecteurs $\vec{Q}$ observés avec leur barre d'erreur, et qui essaye systématiquement toutes les valeurs possibles du vecteur $\vec{k}$ dans la première zone de Brillouin pour indexer les raies magnétiques en appliquant la relation (35).

\section{ON UTILISE LES AUTRES SYMÉTRIES DU CRISTAL: ROTATION ET INVERSION}

Le vecteur de propagation $\overrightarrow{\mathrm{k}}$ est maintenant connu à partir de l'expérience. S'il n'y a qu'un atome magnétique par maille, la seule chose qui reste à déterminer pour connaître complètement la structure magnétique, est la direction de la composante de Fourier $\overrightarrow{\mathrm{m}}^{\mathrm{k}}$. Si, au contraire, il y a plusieurs atomes magnétiques par maille, il faut connaître les orientations relatives des moments de ces différents atomes. En fait, il faut connâtre les composantes $m_{j \alpha}^{k}$ de ces différents atomes qui, dans l'état paramagnétique, au moment de la mise en ordre, minimiseront l'énergie libre : 


$$
\mathrm{U}=-\mathrm{N} \sum_{\mathrm{j}, \mathrm{j}^{\prime}} \sum_{\alpha, \beta} \mathrm{J}_{\mathrm{j} \mathrm{j}^{\prime} \alpha \beta}(\overrightarrow{\mathrm{k}}) \stackrel{\mid}{\mathrm{m}_{\mathrm{j} \alpha}^{\mathrm{k}}} \cdot\left(\mathrm{m}_{\mathrm{j}^{\prime} \beta}^{\overrightarrow{\mathrm{k}}}\right)^{*}
$$

Cette énergie libre s'exprime dans un espace vectoriel sous tendu par les composantes $\mathrm{m}_{\mathbf{j} \alpha}^{\vec{k}}$. Dans cet espace vectoriel, on se propose de faire un changement de vecteurs de base défini par:

$$
\psi_{v}=\sum_{j, \alpha} a_{j \alpha}^{v} m_{j \alpha}^{\bar{k}}
$$

pour obtenir une énergie libre qui s'exprime plus simplement dans cette nouvelle base :

$$
U=-\sum_{w^{\prime}} A_{w^{\prime}}, \psi_{v} \Psi_{v^{*}}
$$

La théorie de Landau va nous aider à trouver les changements de base possibles qui aboutissent à ce que l'énergie libre précédente reste invariable sous laction de toutes les opérations de symétrie du groupe $G_{k}$. Ce groupe $G_{k}$ est le groupe qui comprend parmi toutes les opérations de symétrie du cristal celles qui laissent le vecteur de propagation $\overrightarrow{\mathrm{k}}$ invariant.

La théorie des groupes nous dit qu'on peut construire une énergie libre d'ordre 2 , invariante dans toutes les opérations de symétrie du groupe, à condition de n'utiliser que des vecteurs appartenant à une même représentation irréductible du groupe.

Pour comprendre et utiliser ce résultat, nous allons d'abord expliquer

- ce qu'est un groupe

- ce qu'est une représentation

- ce qu'est une représentation irréductible

\section{DÉFINITION D'UN GROUPE}

Un groupe $G$ est un ensemble d'éléments $A, B, C, \ldots$ avec une loi de composition binaire appelée produit, telle que :

1) le produit de 2 éléments est un élément du groupe : $A B \cup G$

2) le produit est associatif : $A(B C)=(A B) C$

3) il existe un élément identité $\mathrm{E}$ tel que $\mathrm{AE}=\mathrm{EA}=\mathrm{A}$, et cet élément est unique

4) chaque élément a un inverse tel que $\mathrm{AA}^{-1}=\mathbf{A}^{-1} \mathrm{~A}=\mathrm{E}$, et cet inverse est unique

Lordre du groupe g est le nombre d'éléments du groupe

Un exemple : le groupe du triangle équilatéral 


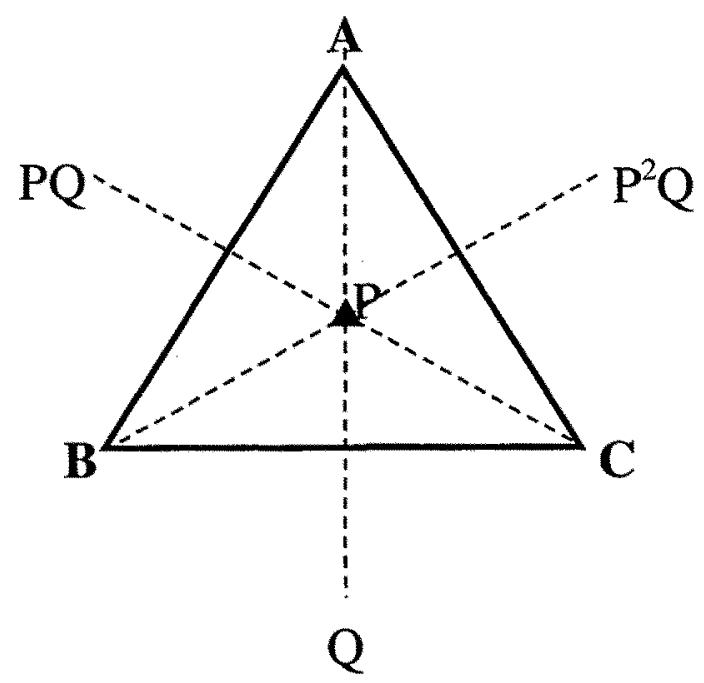

Figure 10: Groupe du triangle équilatéral

\begin{tabular}{|c|c|c|c|c|}
\hline identité, E & $\begin{array}{l}\mathrm{A} \rightarrow \mathrm{A} \\
\mathrm{B} \rightarrow \mathrm{B} \\
\mathrm{C} \rightarrow \mathrm{C}\end{array}$ & $1^{\mathrm{er}}$ axe $3, \mathrm{P}$ & $\begin{array}{l}A \rightarrow B \\
B \rightarrow C \\
C \rightarrow A\end{array}$ & $2^{\text {ème }}$ axe $3, P^{2}$ \\
\hline $1^{\mathrm{cr}}$ miroir, $\mathrm{Q}$ & $\begin{array}{l}A \rightarrow A \\
B \rightarrow C \\
C \rightarrow B\end{array}$ & $2^{\text {ème }}$ miroir, $P Q$ & $\begin{array}{l}A \rightarrow B \\
B \rightarrow A \\
C \rightarrow C\end{array}$ & $3^{\text {ème }}$ miroir, $\mathrm{P}^{2} \mathrm{Q}$ \\
\hline
\end{tabular}

\section{REPRÉSENTATIONS D'UN GROUPE}

Une représentation $\Gamma$ est un ensemble de g matrices $\Gamma(A), \Gamma(B), \Gamma(C)$...telles que

1) $\Gamma(A) . \Gamma(B)=\Gamma(A B)$

2) $\Gamma(E)$ soit une matrice unité (formée uniquement de 1 sur sa diagonale principale)

3) $\Gamma\left(A^{-1}\right)=[\Gamma(A)]^{-1}$

La dimension d'une représentation a est la dimension de l'ensemble des matrices.

Le caractère d'une matrice ou sa trace $\chi(A)$ est la somme de ses éléments diagonaux. En particulier, $\chi(E)$ est égal à $\mathrm{d}$, la dimension de la représentation.

Un exemple: des représentations pour le groupe du triangle équilatéral

Nous allons construire deux représentations à partir de la transformation des coordonnées d'un vecteur polaire $\overrightarrow{\mathbf{r}}$ 
On prend d'abord comme vecteurs de base $\vec{U}$ et $\vec{V}\left\{\begin{array}{l}\vec{U}=\overrightarrow{O B} \\ \vec{V}=\overrightarrow{O C}\end{array}\right.$

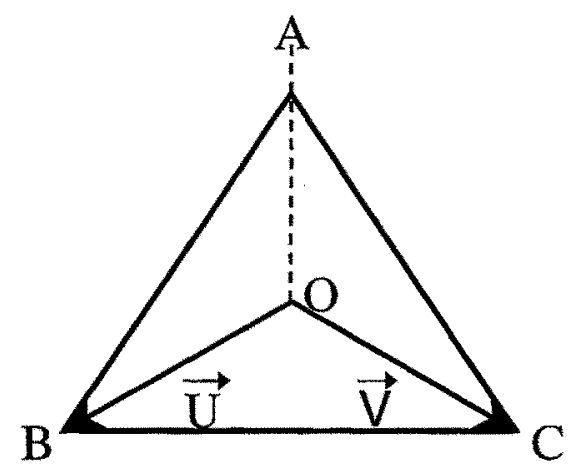

Figure 11 : Vecteurs polaires, 1er choix de la base

ce qui nous donne comme coordonnées pour les 3 vecteurs

$$
\overrightarrow{O A}\left(\begin{array}{l}
-1 \\
-1
\end{array}\right), \quad \overrightarrow{O B}\left(\begin{array}{l}
1 \\
0
\end{array}\right), \quad \overrightarrow{O C}\left(\begin{array}{l}
0 \\
1
\end{array}\right)
$$

Une représentation d'ordre 2 est constituée par l'ensemble des matrices qui transforment les coordonnées $x$, y d'un vecteur $\overrightarrow{\mathrm{r}}=\mathrm{x} \overrightarrow{\mathrm{OA}}+\mathrm{y} \overrightarrow{\mathrm{OB}}$.

\begin{tabular}{|l|l|l|l|l|l|l|l|}
\hline & $\Gamma(\mathrm{E})$ & $\Gamma(\mathrm{P})$ & $\Gamma\left(\mathrm{P}^{2}\right)$ & $\Gamma(\mathrm{Q})$ & $\Gamma(\mathrm{PQ})$ & $\Gamma\left(\mathrm{P}^{2} \mathrm{Q}\right)$ \\
\hline$\Gamma$ & $\left(\begin{array}{ll}1 & 0 \\
0 & 1\end{array}\right)$ & $\left(\begin{array}{cc}0 & -1 \\
1 & -1\end{array}\right)$ & $\left(\begin{array}{rr}-1 & 1 \\
-1 & 0\end{array}\right)$ & $\left(\begin{array}{ll}0 & 1 \\
1 & 0\end{array}\right)$ & $\left(\begin{array}{ll}-1 & 0 \\
-1 & 1\end{array}\right)$ & $\left(\begin{array}{ll}1 & -1 \\
0 & -1\end{array}\right)$ \\
\hline$\chi$ & 2 & -1 & -1 & 0 & 0 & 0 \\
\hline
\end{tabular}

On peut imaginer une autre représentation en prenant d'autres vecteurs de base :

$$
\left\{\begin{array}{l}
\vec{U}=\frac{-\overrightarrow{O B}+\overrightarrow{O C}}{\sqrt{3}} \\
\vec{V}=\overrightarrow{O A}
\end{array}\right.
$$




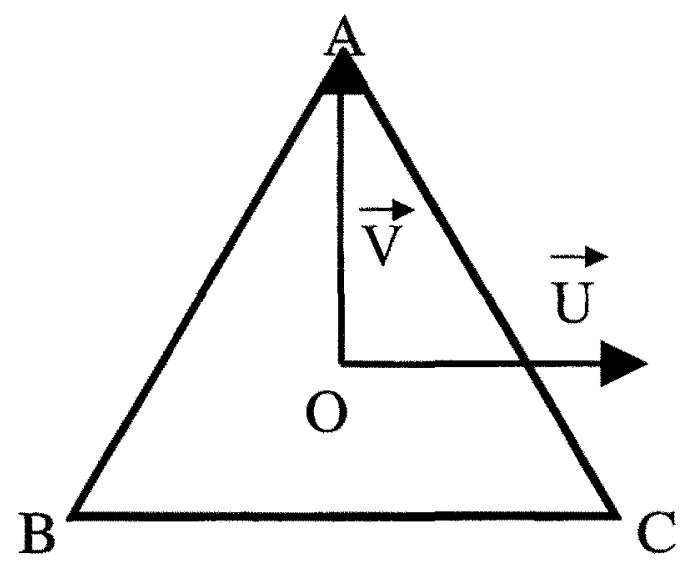

Figure 12 : Vecteurs polaires, $2^{\text {ème }}$ choix de la base

Avec ces nouveaux vecteurs de base, les coordonnées des 3 vecteurs $\overrightarrow{O A}, \overrightarrow{O B}$ et $\overrightarrow{O C}$ deviennent :

$$
\overrightarrow{O A}\left(\begin{array}{l}
0 \\
1
\end{array}\right), \quad \overrightarrow{O B}\left(\begin{array}{c}
-\sqrt{3} / 2 \\
-1 / 2
\end{array}\right), \quad \overrightarrow{O C}\left(\begin{array}{c}
\sqrt{3} / 2 \\
-1 / 2
\end{array}\right)
$$

et les matrices qui représentent les changements de coordonnées deviennent :

\begin{tabular}{|l|l|l|l|l|l|l|l|l|}
\hline & $\Gamma(\mathrm{E})$ & $\Gamma(\mathrm{P})$ & $\Gamma\left(\mathrm{P}^{2}\right)$ & $\Gamma(\mathrm{Q})$ & $\Gamma(\mathrm{PQ})$ & $\Gamma\left(\mathrm{P}^{2} \mathrm{Q}\right)$ \\
\hline$\Gamma$ & $\left(\begin{array}{ll}1 & 0 \\
0 & 1\end{array}\right)$ & $\left(\begin{array}{cc}-1 & -\sqrt{3} \\
\sqrt{3} & -1\end{array}\right)$ & $\left(\begin{array}{cc}-1 & \sqrt{3} \\
-\sqrt{3} & -1\end{array}\right)$ & $\left(\begin{array}{cc}-1 & 0 \\
0 & 1\end{array}\right)$ & $\frac{1}{2}\left(\begin{array}{cc}1 & -\sqrt{3} \\
-\sqrt{3} & -1\end{array}\right)$ & $\frac{1}{2}\left(\begin{array}{cc}1 & \sqrt{3} \\
\sqrt{3} & -1\end{array}\right)$ \\
\hline$\chi$ & 2 & -1 & -1 & 0 & 0 & 0 \\
\hline
\end{tabular}

Il s'agit aussi d'une représentation du groupe, différente de la première mais néanmoins équivalente à elle car elle ne correspond qu'à un changement de vecteurs de base. On a confirmation de cette équivalence en constatant que les caractères de ces deux représentations sont les mêtnes.

\section{VECTEURS POLAIRES ET VECTEURS AXIAUX}

Un vecteur $\vec{r}$ reliant deux points de l'espace est un vecteur polaire (ou vecteur réel)

Un moment magnétique $\overrightarrow{\mathrm{m}}$ est un vecteur axial (ou pseudo vecteur) car il résulte d'une boucle de courant. 


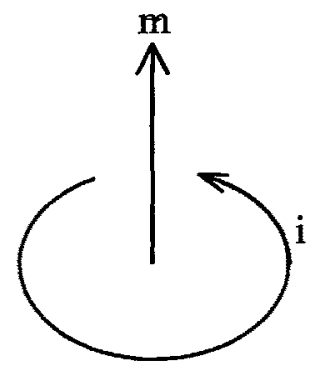

Figure 13 : Vecteur axial résultant d'une boucle de courant

Examinons l'action des opérations de symétrie sur les vecteurs axiaux, en regardant comment elles transforment la boucle de courant:

- une rotation $\mathrm{R}$ fait tourner la boucle de courant donc le vecteur axial

- linversion $\overline{\mathbf{l}}$ n'a aucune action sur une boucle de courant, donc aucune action sur un vecteur axial

- un miroir $\mathrm{m}$ doit être considéré comme une rotation d'axe 2 perpendiculaire au miroir suivie d'une inversion: $\mathrm{m}=\overrightarrow{1} \cdot 2_{\perp}$

l'axe $2_{\perp}$ fait tourner le vecteur axial

l'inversion $\overline{1}$ ne lui fait rien

Si dans l'exemple du groupe du triangle équilatéral on considère, au lieu des 3 vecteurs polaires $\overrightarrow{O A}, \overrightarrow{O B}$ et $\overrightarrow{O C}$ les 3 vecteurs axiaux $\vec{S}_{A}, \vec{S}_{B}$ et $\vec{S}_{C}$, l'action des opérateurs de symétrie du groupe devient:

$$
\begin{array}{lll}
\mathrm{P} \overrightarrow{\mathrm{S}}_{\mathrm{A}}=\overrightarrow{\mathrm{S}}_{\mathrm{B}} & \mathrm{P}^{2} \overrightarrow{\mathrm{S}}_{\mathrm{A}}=\overrightarrow{\mathrm{S}}_{\mathrm{C}} & \mathrm{Q} \overrightarrow{\mathrm{S}}_{\mathrm{A}}=\overline{1} .2_{\perp} \overrightarrow{\mathrm{S}}_{\mathrm{A}}=-\overrightarrow{\mathrm{S}}_{\mathrm{A}} \\
\mathrm{P} \overrightarrow{\mathrm{S}}_{\mathrm{B}}=\overrightarrow{\mathrm{S}}_{\mathrm{C}} & \mathrm{P}^{2} \overrightarrow{\mathrm{S}}_{\mathrm{B}}=\overrightarrow{\mathrm{S}}_{\mathrm{A}} & \mathrm{Q} \overrightarrow{\mathrm{S}}_{\mathrm{B}}=\overline{1} .2_{\perp} \overrightarrow{\mathrm{S}}_{\mathrm{B}}=-\overrightarrow{\mathrm{S}}_{\mathrm{C}} \\
\mathrm{P} \overrightarrow{\mathrm{S}}_{\mathrm{C}}=\overrightarrow{\mathrm{S}}_{\mathrm{A}} & \mathrm{P}^{2} \overrightarrow{\mathrm{S}}_{\mathrm{C}}=\overrightarrow{\mathrm{S}}_{\mathrm{B}} & \mathrm{Q} \overrightarrow{\mathrm{S}}_{\mathrm{C}}=\overline{1} .2_{\perp} \overrightarrow{\mathrm{S}}_{\mathrm{C}}=-\overrightarrow{\mathrm{S}}_{\mathrm{B}}
\end{array}
$$

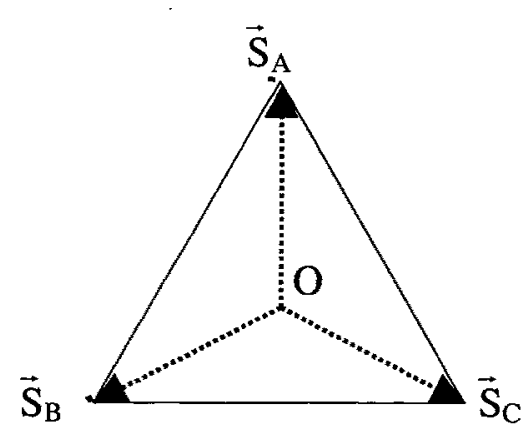

Figure 14 : Vecteurs axiaux.

Si on prend comme nouveaux vecteurs de base : $\left\{\begin{array}{l}\vec{U}=\overrightarrow{S_{B}} \\ \vec{V}=\vec{S}_{C}\end{array}\right.$

la représentation déduite de la transformée des coordonnées des vecteurs axiaux est : 


\begin{tabular}{|l|l|l|l|l|l|l|l|}
\hline & $\Gamma(\mathrm{E})$ & $\Gamma(\mathrm{P})$ & $\Gamma\left(\mathrm{P}^{2}\right)$ & $\Gamma(\mathrm{Q})$ & $\Gamma(\mathrm{PQ})$ & $\Gamma\left(\mathrm{P}^{2} \mathrm{Q}\right)$ \\
\hline$\Gamma$ & $\left(\begin{array}{ll}1 & 0 \\
0 & 1\end{array}\right)$ & $\left(\begin{array}{ll}0 & -1 \\
1 & -1\end{array}\right)$ & $\left(\begin{array}{ll}-1 & 1 \\
-1 & 0\end{array}\right)$ & $\left(\begin{array}{cc}0 & -1 \\
-1 & 0\end{array}\right)$ & $\left(\begin{array}{cc}1 & 0 \\
1 & -1\end{array}\right)$ & $\left(\begin{array}{cc}-1 & 1 \\
0 & 1\end{array}\right)$ \\
\hline$\chi$ & 2 & -1 & -1 & 0 & 0 & 0 \\
\hline
\end{tabular}

Il s'agit ici d'une représentation équivalente à celle obtenue par la transformée des coordonnées des vecteurs polaires, mais cette équivalence est dans le cas présent fortuite et ne correspond pas à une règle générale.

\section{RÉDUCTION D'UNE REPRÉSENTATION, REPRÉSENTATIONS IRRÉDUCTIBLES}

Un changement de vecteurs de base peut amener toutes les matrices d'une représentation à avoir la même structure diagonale en blocs.

$$
\Gamma(\mathrm{A})=\left(\begin{array}{c|c|c}
\tau_{1}(\mathrm{~A}) & 0 & 0 \\
\hline 0 & \tau_{2}(\mathrm{~A}) & 0 \\
\hline 0 & 0 & \tau_{3}(\mathrm{~A})
\end{array}\right), \quad \Gamma(\mathrm{B})=\left(\begin{array}{c|c|c}
\tau_{1}(\mathrm{~B}) & 0 & 0 \\
\hline 0 & \tau_{2}(\mathrm{~B}) & 0 \\
\hline 0 & 0 & \tau_{3}(\mathrm{~B})
\end{array}\right)
$$

On dit alors qu'on a réduit la représentation $\Gamma$ en une somme de représentations :

$$
\Gamma=\tau_{1}+\tau_{2}+\tau_{3}
$$

Les représentations irréductibles sont celles qu'aucun changement de vecteur de base ne peut mettre sous une forme de blocs diagonaux. Bien entendu, les représentations d'ordre 1 sont irréductibles.

II existe un critère pour savoir si une représentation est irréductible. Une représentation est irréductible si :

$$
\frac{1}{\mathrm{~g}} \sum_{\mathrm{G}_{i}} \mid \chi\left(\mathrm{G}_{i}\right)^{2}=1
$$

où g est l'ordre du groupe et où la somme se fait sur tous les éléments du groupe.

Les représentations irréductibles jouent un grand rôle dans la théorie des groupes et nous allons les utiliser.

\section{Réduction d'une représentation en représentations irréductibles}

On peut toujours réduire une représentation $\Gamma$ non irréductible en représentations irréductibles $\tau_{v}$ :

$$
\Gamma=\sum_{v} \mathrm{a}_{v} \tau_{v}
$$


par exemple : $\Gamma=\tau_{1}+\tau_{1}+\tau_{1}+\tau_{2}+\tau_{3}+\tau_{3}=3 \tau_{1}+\tau_{2}+2 \tau_{1}$

Les coefficients $a_{v}$ de cette décomposition sont donnés par:

$$
\mathrm{a}_{v}=\frac{1}{\mathrm{~g}} \sum_{\mathrm{G}_{\mathrm{i}}} \chi_{\mathrm{r}}\left(\mathrm{G}_{\mathrm{j}}\right) \cdot \chi_{\tau_{v}}^{*}\left(\mathrm{G}_{\mathrm{i}}\right)
$$

où la somme se fait sur tous les éléments du groupe.

\section{Recherche des vecteurs de base des représentations irréductibles}

Le principe en est simple. On prend un vecteur dans l'espace de départ et on le projette dans l'espace de la représentation irréductible qui nous intéresse.

L'opérateur qui permet cette projection sur l'espace $\tau_{v}$ est l'opérateur de projection $\mathrm{P}^{v}$ qui s'écrit simplement pour des représentations irréductibles à une dimension :

$$
P^{v}=\sum_{G_{i}} \tau_{v}^{*}\left(G_{i}\right) \cdot G_{i}
$$

et de façon un peu plus compliquée pour des représentations irréductibles à plusieurs dimensions :

$$
\mathrm{P}_{\lambda}^{v}=\sum_{\mathrm{G}_{i}}\left[\tau_{v}\left(\mathrm{G}_{\mathrm{i}}\right)\right]_{\lambda \mu}^{*} \cdot \mathrm{G}_{\mathrm{i}}
$$

On obtient les différents vecteurs de base dans ce demier cas soit en fixant $\mu$ et en faisant varier $\lambda$, soit en faisant le contraire.

\section{GROUPE D'ESPACE ET GROUPE DU VECTEUR $\vec{k}$}

Le groupe d'espace est le groupe des opérateurs de symétrie qui laissent le cristal invariant (sa partie nucléaire).

Les opérateurs en question s'écrivent :

$$
(R \mid \vec{v}) \vec{l}
$$

où $\vec{i}$ est une translation du réseau

où $\vec{v}$ est une translation fractionnaire, interne à la maille

où $R$ est une rotation ou une inversion ou un produit des deux

Les représentations irréductibles du groupe de translations du réseau

- sont de dimension 1

- sont caractérisées par un vecteur $\vec{k}$

$$
\Gamma(\vec{i})=e^{-i \vec{k} \vec{i}}
$$

Le groupe du vecteur $\vec{k}$ ou groupe $G_{k}$ 
C'est le groupe formé par l'ensemble des éléments du groupe d'espace qui, non seulement laissent les atomes du cristal inchangés, mais aussi laissent le vecteur $\overrightarrow{\mathrm{k}}$ inchangé.

Attention, contrairement aux moments magnétiques $\overrightarrow{\mathrm{m}}$ qui sont des vecteurs axiaux, $\overrightarrow{\mathrm{k}}$ est un vecteur polaire: $\overrightarrow{1} \cdot \vec{k}=-\vec{k}$

\section{RECHERCHE DES STRUCTURES MAGNÉTIQUES POSSIBLES}

1) On détermine expérimentalement, à partir de diagrammes de poudres, le vecteur de propagation $\overrightarrow{\mathrm{k}}$ de la structure.

2) On sélectionne dans le groupe d'espace $G$ tous les éléments qui laissent $\vec{k}$ inchangé. On arrive ainsi au groupe $\mathrm{G}_{\mathrm{k}}$.

On vérifie les éléments du groupe $\mathrm{G}_{\mathrm{k}}$ dans Kovalev, "Irreducible Representations of Space Groups", Gordon and Breach, 1965

ou dans Bradley, Cracknell, "The Mathematical Theory of Symmetry inSolids", Clarendon Press, 1972

3) D'une part, on relève dans un de ces deux ouvrages les représentations irréductibles $\tau_{v}$ du groupe $\mathrm{G}_{\mathrm{k}}$.

Attention: Sont tabulées les représentations $\hat{\tau}_{v}$ dites pondérées ou "loaded". Pour obtenir les vraies représentations, il faut écrire :

$$
\tau_{v}=\hat{\tau}_{v} \mathrm{e}^{-\mathrm{i} \hat{k}_{v}}
$$

où $\vec{v}$ représente la translation fractionnaire, inteme à la maille, de chaque opérateur de symétrie.

4) D'autre part, on écrit la représentation vectorielle axiale $\Gamma\left(G_{k}\right)$ des moments magnétiques qui indique comment les différents opérateurs du groupe $G_{k}$ transforment chacune des composantes $\mathrm{m}_{\mathrm{j} \alpha}^{\mathrm{k}}$ des différents moments magnétiques de la maille élémentaire, ou, plus exactement, de ses composantes de Fourier. S'il y a n atomes magnétiques dans la maille élémentaire, la représentation $\Gamma\left(\mathrm{G}_{\mathrm{k}}\right)$ sera d'ordre $3 \mathrm{n}$.

5) On réduit la représentation $\Gamma\left(G_{k}\right)$ en représentations irréductibles $\tau_{v}$ du groupe $G_{k}$

$$
\Gamma=\sum_{v} a_{v} \tau_{v}
$$

en utilisant : $\quad \mathrm{a}_{v}=\frac{1}{\mathrm{~g}} \sum_{\mathrm{G}_{i}} \chi_{\Gamma}\left(\mathrm{G}_{\mathrm{i}}\right) \cdot \chi_{\mathrm{r}_{v}}^{*}\left(\mathrm{G}_{\mathrm{i}}\right)$

6) Pour chaque représentation irréductible $\tau_{y}$ qui se trouve dans la décomposition, on recherche les vecteurs de base $\psi_{v}^{1}, \psi_{v}^{2}, \ldots$ qui sous tendent cet espace. 
Pour cela on utilise l'opérateur de projection $\mathrm{P}^{v}=\sum_{\mathrm{G}_{\mathrm{ki}}} \tau_{v}^{*}\left(\mathrm{G}_{\mathrm{ki}}\right) \cdot \mathrm{G}_{\mathrm{ki}}$ que l'on applique successivement à $\mathrm{m}_{1 \mathrm{x}}^{\mathrm{k}}, \mathrm{m}_{1 \mathrm{y}}^{\mathrm{k}}, \mathrm{m}_{1 \mathrm{z}}^{\mathrm{k}}, \mathrm{m}_{2 \mathrm{x}}^{\mathrm{k}}, \ldots$.

7) On utilise d'une part la théorie de Landau qui dit que la structure magnétique qui s'établit au moment d'une transition du $2^{\text {ème }}$ ordre correspond à un mode magnétique qui persiste alors que les autres s'annulent et d'autre part la théorie des groupes qui permet de déterminer les modes à partir des vecteurs de base des représentations irréductibles (pour obtenir une énergie libre invariante dans les opérations du groupe $G_{k}$ ).

Les structures magnétiques compatibles avec la représentation irréductible $\tau_{1}$ sont celles où les vecteurs $\psi_{1}^{n}$ existent alors que tous les autres vecteurs $\psi_{v}^{n}$ sont égaux à zéro.

Les structures magnétiques compatibles avec la représentation irréductible $\tau_{2}$ sont celles où les vecteurs $\psi_{2}^{n}$ existent alors que tous les autres vecteurs $\psi_{v}^{n}$ sont égaux à zéro.

Et ainsi de suite...

8) On teste, à partir des intensités des raies magnétiques, laquelle (ou lesquelles) des structures magnétiques possibles est compatible avec l'expérience et on détermine les paramètres laissés libres par la théorie.

\section{UN EXEMPLE: LA STRUCTURE MAGNÉTIQUE DE CeAl 2}

$\mathrm{CeAl}_{2}$ est un composé cubique à faces centrées (figure 15) qui cristallise dans le groupe d'espace $\mathrm{Fd} 3 \mathrm{~m}$ ou $\mathrm{O}_{\mathrm{h}}^{7}\left(\mathrm{n}^{\circ} 227\right.$ des tables internationales) avec 2 atomes de Ce dans la maille élémentaire: en $(0,0,0)$ et en $(1 / 4,1 / 4,1 / 4)$ si on utilise la description non centrée du groupe d'espace.

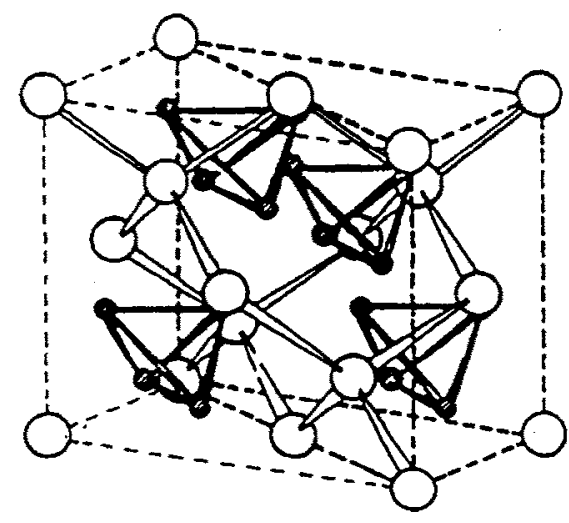

Atome de $\mathrm{Ce}$

Atome d'Al

Cel $(0,0,0)$

$\mathrm{Ce} 2(1 / 4,1 / 4,1 / 4)$

Figure 15 : Structure de $\mathrm{CeAl}_{2}$ * 
Les diagrammes de poudre mesurés à $1.9 \mathrm{~K}$ et à $8.2 \mathrm{~K}$, c'est à dire au dessous et au dessus de la température d'ordre $\left(\mathrm{T}_{\mathrm{N}}=3.8 \mathrm{~K}\right)$, ainsi que la différence entre ces deux diagrammes sont représentés sur la figure 16. On voit bien sur cette différence l'apparition des réflexions magnétiques qui ont pu être indexées avec un vecteur de propagation $\overrightarrow{\mathrm{k}}=\left(\frac{1}{2}-\delta, \frac{1}{2}+\delta, \frac{1}{2}\right)$, vecteur dont l'extrémité est située sur la surface de la zone de Brillouin.
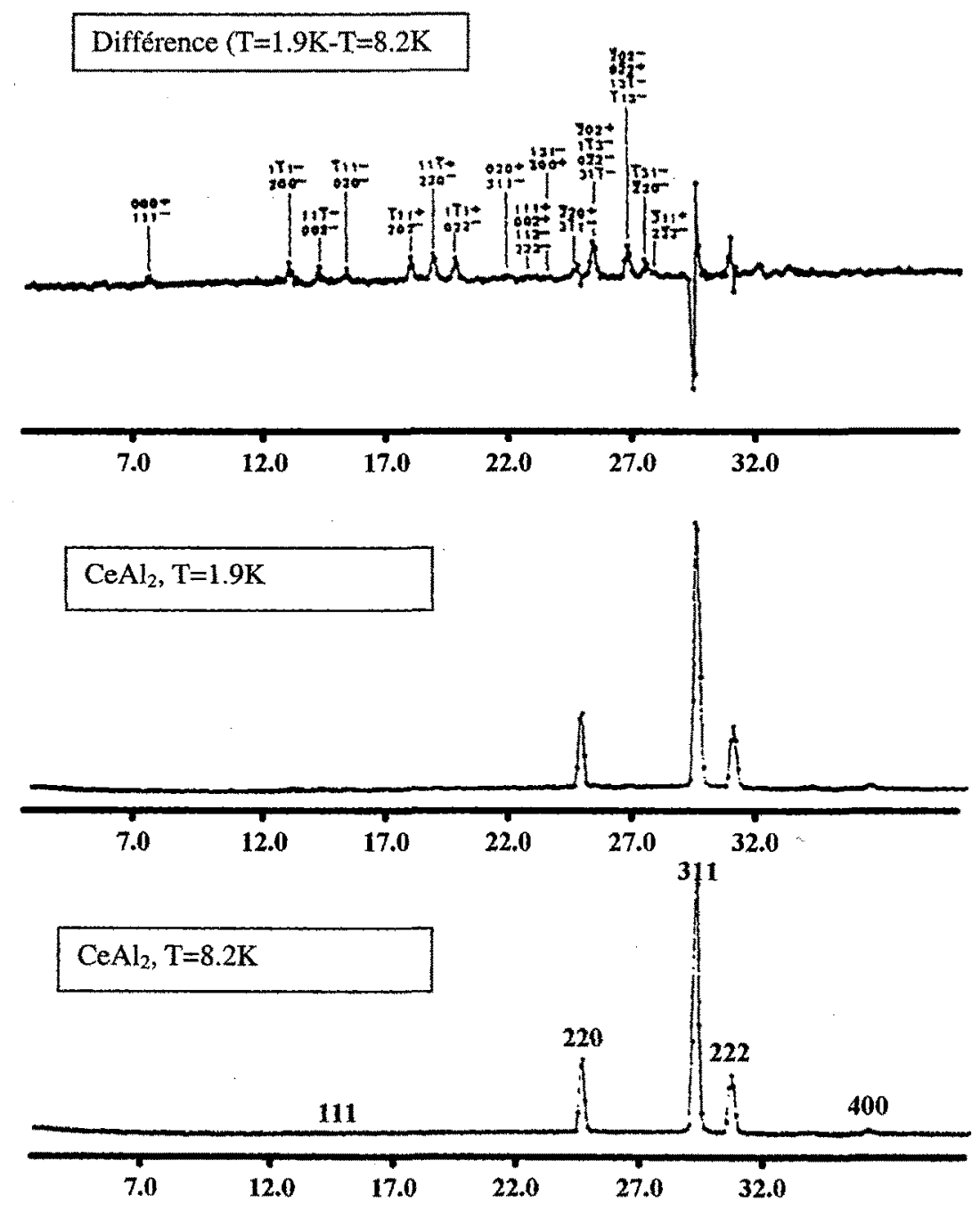

Figure 16: Diagrammes de poudres du $\mathrm{Ce} \mathrm{Al}_{2}$ de part et d'autre de la transition magnétique. 
Des 48 opérateurs qui forment le groupe $\mathrm{G}$ (opérateurs de translation de réseau exclus), seuls 2 d'entre eux laissent le vecteur $\vec{k}$ inchangé, ce sont l'identité $h_{1}$ (notations de Kovalev) qui transforme les coordonnées $(x, y, z)$ en $(x, y, z)$ et $h_{13}$, un axe de rotation d'ordre 2 qui transforme les coordonnées $(x, y, z)$ en $(1 / 4-y, 1 / 4-x, 1 / 4-z)$ (voir figure 17). Ces deux opérateurs forment le groupe $\mathrm{G}_{\mathrm{k}}$.

\section{Groupe : Fd3m}

No. 227

$F 4_{1} / d \overline{3} 2 / m$

$$
O_{h}^{7}
$$

Origin at $\overline{4} 3 m$, at $\frac{\overline{1}}{8}, \frac{\overline{1}}{8}, \frac{\overline{1}}{8}$ from centre $((\overline{3} m))$

Nurnber cf positions,

Wyckoff notation, and point symmetry

\section{Co-ordînates of equivalent positions}

$$
\left(0,0,0 ; \quad 0, \frac{1}{2}, \frac{1}{2} ; \quad \frac{1}{2}, 0, \frac{1}{2} ; \quad \frac{1}{2}, \frac{1}{2}, 0\right)+
$$

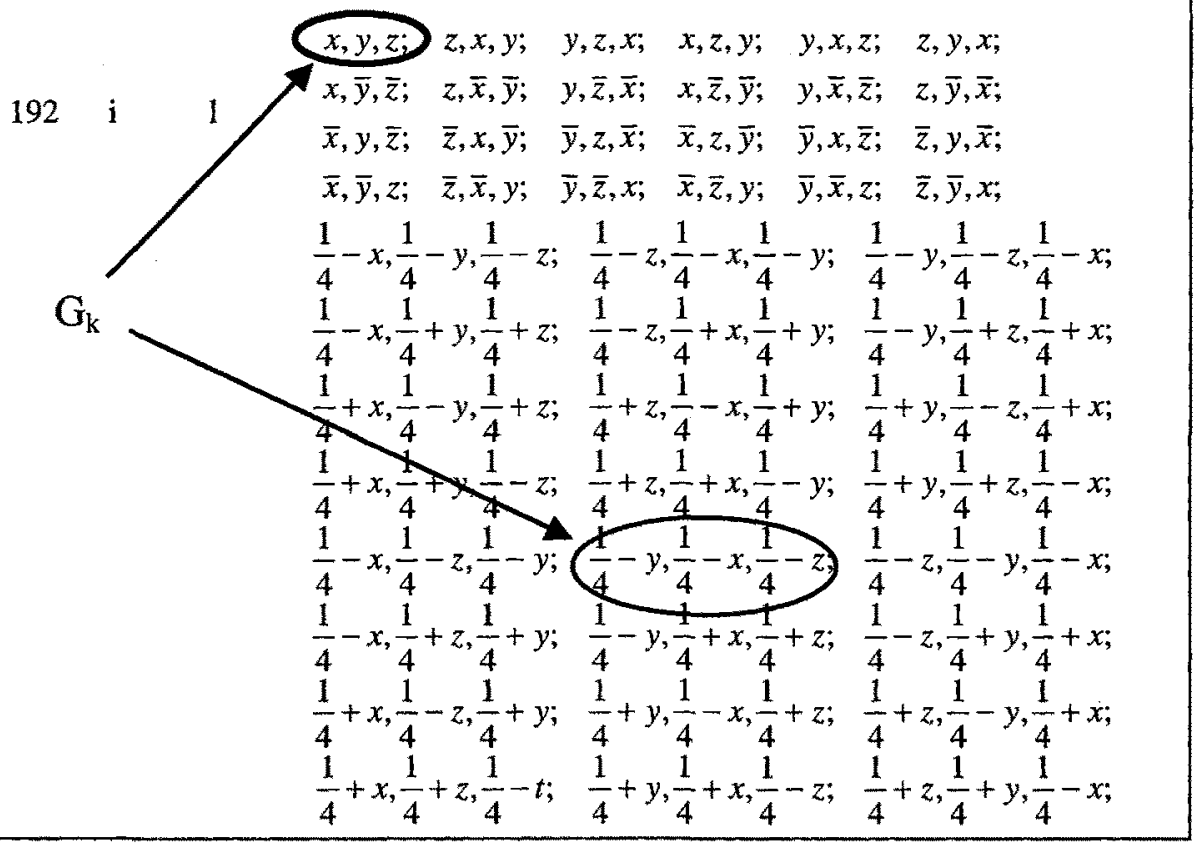

Figure 17 : Groupe $G$ et groupe $G_{k}$, tables Internationales, description non centro

En vérifiant dans Kovalev, page 60, (figure 18) on voit que dans les réseaux cubique à faces centrées $\left(\Gamma_{\mathrm{c}}^{\mathrm{f}}\right)$, notre vecteur $\overrightarrow{\mathrm{k}}=\left(\frac{1}{2}-\delta, \frac{1}{2}+\delta, \frac{1}{2}\right)$ est libellé $\overrightarrow{\mathrm{k}}_{3}$. Le tableau du groupe $\mathrm{O}_{\mathrm{h}}^{7}$ (Kovalev page 62, figure 18) nous indique que pour ce groupe d'espace et pour un vecteur de type $\overrightarrow{\mathrm{k}}_{3}$, les représentations irréductibles sont données dans le tableau T218. Ce tableau T218 se trouve à la page 97 de Kovalev (figure 18) et utilise la notation $\sigma=\mathrm{e}^{\frac{i \pi}{4}}$. En fait il ne donne pas les 
représentations irréductibles, mais donne les représentations irréductibles pondérées: elles sont au nombre de deux et sont dordre un.

\section{Kovalev, page 60}

12. Cubic system, lattice $\Gamma_{c}^{f}$

The vectors in the inverse lattice are equal

$\vec{b}_{1}=\left(-\frac{\pi}{\tau}, \frac{\pi}{\tau}, \frac{\pi}{\tau}\right), \vec{b}_{2}=\left(\frac{\pi}{\tau},-\frac{\pi}{\tau}, \frac{\pi}{\tau}\right), \vec{b}_{1}=\left(\frac{\pi}{\tau}, \frac{\pi}{\tau},-\frac{\pi}{\tau}\right)$.

\begin{tabular}{|l|l|l|}
\hline $\begin{array}{l}\text { Vectors } \vec{k}_{l} \text { as linear } \\
\text { combinations of the vectors } \\
\vec{b}_{1}, \vec{b}_{2}, \vec{b}_{3}\end{array}$ & $\begin{array}{l}\text { Vectors } \vec{k}_{l} \text { in terms of } \\
\text { projections on the } k_{x}, k_{y} \\
\text { and } k_{z} \text { axes. }\end{array}$ & $\begin{array}{l}\text { Elements } h_{l} \text { in the class } O_{h} \\
\text { that leave the vector } \vec{k}_{i} \\
\text { Fixed or change it by a vector } \\
\text { in the inverse lattice }\end{array}$ \\
\hline$\vec{k}_{1}=\mu\left(\vec{b}_{1}+\vec{b}_{3}\right)+\mu_{1}\left(\vec{b}_{2}+\vec{b}_{3}\right)$ & $\frac{2 \mu_{1} \pi}{\tau}, \frac{2 \mu \pi}{\tau}, 0$ & $h_{1}, h_{28}$ \\
\hline$\vec{k}_{2}=\mu\left(\vec{b}_{1}+\vec{b}_{2}\right)+\mu_{3}\left(\vec{b}_{3}\right)$ & $\frac{\mu_{3} \pi}{\tau}, \frac{\mu_{3} \pi}{\tau}, \frac{\left(2 \mu-\mu_{3}\right) \pi}{\tau}$ & $h_{1}, h_{37}$ \\
\hline$\vec{k}_{3}=\mu\left(\vec{b}_{1}-\vec{b}_{2}\right)+\frac{1}{2}\left(\vec{b}_{1}+\vec{b}_{2}+\vec{b}_{3}\right)$ & $\frac{(1-4 \mu) \pi}{2 \tau}, \frac{(1+4 \mu) \pi}{2 \tau}, \frac{\pi}{2 \tau}$ & $h_{1}, h_{13}$ \\
\hline
\end{tabular}

\section{Kovalev, page 62}

Group $O_{h}^{7}$

$\vec{k}_{3}-2 . \quad \vec{k}_{2}-118 . \quad \vec{k}_{3}-218 . \quad \vec{k}_{4}-146 . \quad \vec{k}_{5}-191 . \quad \vec{k}_{6}-119$.

$\vec{k}_{7}-44 . \quad \vec{k}_{8}-224 . \quad \vec{k}_{9}-225 . \quad \vec{k}_{10}-159 . \quad \vec{k}_{11}-205$.

\section{Kovalev, page 97}

\begin{tabular}{|l|l|l|}
\hline $\mathrm{T} 218$ & $h_{l}$ & $h_{13}$ \\
\hline$\hat{t}^{1}$ & 1 & $\sigma^{3}$ \\
\hline$\hat{t}^{2}$ & 1 & $\sigma^{7}$ \\
\hline
\end{tabular}

Figure 18: Extraits de Kovalev
Compte tenu que

$$
\text { pour } h_{1}
$$$$
\overrightarrow{\mathrm{k}} \cdot \overrightarrow{\mathrm{v}}=0
$$$$
\text { pour } h_{13}
$$$$
\overrightarrow{\mathrm{k}} \cdot \overrightarrow{\mathrm{v}}=2 \pi\left(\frac{1}{2} \cdot \frac{1}{4}+\frac{1}{2} \cdot \frac{1}{4}+\frac{1}{2} \cdot \frac{1}{4}\right)=\frac{3 \pi}{4}
$$

les deux représentations irréductibles (non pondérées cette fois) du groupe $G_{k}$ sont données (formule 47) dans le tableau suivant : 


\begin{tabular}{|l|l|l|}
\hline & $h_{1}$ & $h_{2}$ \\
\hline$\tau_{1}$ & 1 & 1 \\
\hline$\tau_{2}$ & 1 & -1 \\
\hline
\end{tabular}

Parallèlement, nous écrivons la représentation vectorielle axiale $\Gamma(\mathrm{h})$ des moments magnétiques. Pour celà, nous commençons par examiner laction des deux opérateurs $h_{1}$ et $h_{13}$ sur les 6 composantes $\mathrm{m}_{\mathrm{ix}}^{\mathrm{k}}, \mathrm{m}_{1 y}^{\mathrm{k}}, \mathrm{m}_{1 \mathrm{z}}^{\mathrm{k}}, \mathrm{m}_{2 \mathrm{x}}^{\mathrm{k}}, \mathrm{m}_{2 y}^{\mathrm{k}}$ et $\mathrm{m}_{2 \mathrm{z}}^{\mathrm{k}}$ des deux moments magnétiques, ou plutôt de leurs composantes de Fourier $\overrightarrow{\mathrm{m}}_{\mathrm{Ce} 1}^{\mathrm{k}}$ et $\overrightarrow{\mathrm{m}}_{\mathrm{Ce} 2}^{\mathrm{k}}$, en n'oubliant pas qu'il s'agit de vecteurs axiaux (pseudo vecteurs).

\begin{tabular}{|c|cc|}
\hline $\begin{array}{c}\text { composantes } \\
\text { de départ }\end{array}$ & $\mathrm{h}_{1}$ & $\mathrm{~h}_{13}$ \\
$(\mathrm{x}, \mathrm{y}, \mathrm{z})$ & $\left(\frac{1}{4}-\mathrm{y}, \frac{1}{4}-\mathrm{x}, \frac{1}{4}-\mathrm{z}\right)$ \\
\hline $\mathrm{m}_{1 \mathrm{x}}^{\mathrm{k}}$ & $\mathrm{m}_{1 \mathrm{x}}^{\mathrm{k}}$ & $-\mathrm{m}_{2 \mathrm{y}}^{\mathrm{k}}$ \\
$\mathrm{m}_{1 \mathrm{y}}^{\mathrm{k}}$ & $\mathrm{m}_{1 y}^{\mathrm{k}}$ & $-\mathrm{m}_{2 \mathrm{x}}^{\mathrm{k}}$ \\
$\mathrm{m}_{1 z}^{\mathrm{k}}$ & $\mathrm{m}_{1 z}^{\mathrm{k}}$ & $-\mathrm{m}_{2 z}^{\mathrm{k}}$ \\
$\mathrm{m}_{2 \mathrm{x}}^{\mathrm{k}}$ & $\mathrm{m}_{2 \mathrm{x}}^{\mathrm{k}}$ & $-\mathrm{m}_{1 \mathrm{y}}^{\mathrm{k}}$ \\
$\mathrm{m}_{2 \mathrm{y}}^{\mathrm{k}}$ & $\mathrm{m}_{2 \mathrm{y}}^{\mathrm{k}}$ & $-\mathrm{m}_{1 \mathrm{x}}^{\mathrm{k}}$ \\
$\mathrm{m}_{2 z}^{\mathrm{k}}$ & $\mathrm{m}_{2 z}^{\mathrm{k}}$ & $-\mathrm{m}_{1 z}^{\mathrm{k}}$ \\
\hline
\end{tabular}

La représentation $\Gamma(h)$, ainsi que les caractères correspondant sont donnés dans le tableau suivant :

\begin{tabular}{|c|c|c|c|c|c|c|c|c|c|c|c|c|}
\hline & \multicolumn{6}{|c|}{$h_{1}$} & \multicolumn{6}{|c|}{$\mathrm{h}_{13}$} \\
\hline \multirow{6}{*}{$\Gamma$} & $(1$ & 0 & 0 & 0 & 0 & 0 & 0 & 0 & 0 & 0 & -1 & 0 \\
\hline & 0 & 1 & 0 & 0 & 0 & 0 & 0 & 0 & 0 & -1 & 0 & 0 \\
\hline & 0 & 0 & 1 & 0 & 0 & c & 0 & 0 & 0 & 0 & 0 & -1 \\
\hline & 0 & 0 & 0 & 1 & 0 & 0 & 0 & -1 & 0 & 0 & 0 & 0 \\
\hline & 0 & 0 & 0 & 0 & 1 & 0 & -1 & 0 & 0 & 0 & 0 & 0 \\
\hline & 0 & 0 & 0 & 0 & 0 & 1 & 0 & 0 & -1 & 0 & 0 & 0 \\
\hline$x$ & \multicolumn{6}{|c|}{6} & \multicolumn{6}{|c|}{0} \\
\hline
\end{tabular}

Cette représentation est réductible. En effet, le critère de réducibilité (40) donne :

$$
\frac{1}{\mathrm{~g}} \sum_{\mathrm{h}_{\mathrm{i}}} \mid \chi\left(\mathrm{h}_{\mathrm{i}}\right)^{2}=\frac{1}{2}(36+0)=18 \neq 1
$$

La décomposition en représentations irréductibles $\tau_{1}$ et $\tau_{2}$ est donné par l'application de la formule (42) 


$$
\begin{aligned}
& \mathrm{a}_{1}=\frac{1}{\mathrm{~g}} \sum_{\mathrm{h}_{\mathrm{i}}} \chi_{\Gamma}\left(\mathrm{h}_{\mathrm{i}}\right) \cdot \chi_{\tau_{\mathrm{i}}}^{*}\left(\mathrm{~h}_{\mathrm{i}}\right)=\frac{1}{2}(6+0)=3 \\
& \mathrm{a}_{2}=\frac{1}{\mathrm{~g}} \sum_{\mathrm{h}_{\mathrm{i}}} \chi_{\Gamma}\left(\mathrm{h}_{\mathrm{i}}\right) \cdot \chi_{\tau_{2}}^{*}\left(\mathrm{~h}_{\mathrm{i}}\right)=\frac{1}{2}(6+0)=3
\end{aligned}
$$

Ce qui donne :

$$
\Gamma=3 \tau_{1}+3 \tau_{2}
$$

Il ne nous reste qu'à trouver les vecteurs de base des deux sous espaces $\tau_{1}$ et $\tau_{2}$. Pour cela nous utilisons les deux opérateurs de projection donnés par la formule (43):

$$
\begin{aligned}
& P^{1}=\sum_{h_{i}} \tau_{1}^{*}\left(h_{i}\right) \cdot h_{i}=h_{1}+h_{13} \\
& P^{2}=\sum_{h_{i}} \tau_{2}^{*}\left(h_{i}\right) \cdot h_{i}=h_{1}-h_{13}
\end{aligned}
$$

\section{Ce qui nous donne, pour la représentation $\tau_{1}$ :}

$$
\left.\begin{array}{rl}
\mathrm{P}^{1} \mathrm{~m}_{\mathrm{tx}}^{\mathrm{k}}=\mathrm{m}_{1 \mathrm{x}}^{\mathrm{k}}-\mathrm{m}_{2 \mathrm{y}}^{\mathrm{k}}=\psi_{1}^{\mathrm{1}} \\
\mathrm{P}^{1} \mathrm{~m}_{1 \mathrm{y}}^{\mathrm{k}}=\mathrm{m}_{1 \mathrm{y}}^{\mathrm{k}}-\mathrm{m}_{2 \mathrm{x}}^{\mathrm{k}}=\psi_{1}^{2} \\
\mathbf{P}^{1} \mathrm{~m}_{1 z}^{\mathrm{k}}=\mathrm{m}_{1 z}^{\mathrm{k}}-\mathrm{m}_{2 z}^{\mathrm{k}}=\psi_{1}^{3}
\end{array}\right\} 3 \text { vecteurs de } \tau_{1}
$$

et accessoirement, si on applique $\mathrm{P}^{1}$ sur d'autres composantes, on obtient les mêmes vecteurs, à un coefficient près :

$$
\begin{aligned}
& \mathrm{P}^{1} \mathrm{~m}_{2 \mathrm{x}}^{\mathrm{k}}=\mathrm{m}_{2 \mathrm{x}}^{\mathrm{k}}-\mathrm{m}_{1 \mathrm{y}}^{\mathrm{k}}=-\psi_{1}^{2} \\
& \mathbf{P}^{1} \mathrm{~m}_{2 \mathrm{y}}^{\mathrm{k}}=\mathrm{m}_{2 \mathrm{y}}^{\mathrm{k}}-\mathrm{m}_{1 \mathrm{x}}^{\mathrm{k}}=-\psi_{1}^{1} \\
& \mathrm{P}^{\mathrm{l}} \mathrm{m}_{2 \mathrm{z}}^{\mathrm{k}}=\mathrm{m}_{2 \mathrm{z}}^{\mathrm{k}}-\mathrm{m}_{1 \mathrm{z}}^{\mathrm{k}}=-\psi_{1}^{3}
\end{aligned}
$$

\section{Pour la représentation $\tau_{2}$ :}

$$
\left.\begin{array}{rl}
\mathrm{P}^{2} \mathrm{~m}_{1 \mathrm{x}}^{\mathrm{k}}=\mathrm{m}_{1 \mathrm{x}}^{\mathrm{k}}+\mathrm{m}_{2 y}^{\mathrm{k}}=\psi_{2}^{1} \\
\mathrm{P}^{2} \mathrm{~m}_{1 \mathrm{y}}^{\mathrm{k}}=\mathrm{m}_{1 \mathrm{y}}^{\mathrm{k}}+\mathrm{m}_{2 \mathrm{x}}^{\mathrm{k}}=\psi_{2}^{2} \\
\mathrm{P}^{2} \mathrm{~m}_{1 \mathrm{z}}^{\mathrm{k}}=\mathrm{m}_{1 \mathrm{x}}^{\mathrm{k}}+\mathrm{m}_{2 \mathrm{x}}^{\mathrm{k}}=\psi_{2}^{3}
\end{array}\right\} 3 \text { vecteurs de } \tau_{2}
$$

\section{Il y a donc deux structures magnétiques possibles.}

Pour la représentation $\tau_{1}$, une structure antiferromagnétique, à priori non colinéaire, avec 3 paramètres à affiner :

$$
\begin{array}{ll}
\mathrm{m}_{1 \mathrm{x}}^{\mathrm{k}}-\mathrm{m}_{2 \mathrm{y}}^{\mathrm{k}}=\mathrm{a} & \text { mais } \mathrm{m}_{1 \mathrm{x}}^{\mathrm{k}}+\mathrm{m}_{2 \mathrm{y}}^{\mathrm{k}}=0 \Rightarrow \mathrm{m}_{2 \mathrm{y}}^{\mathrm{k}}=-\mathrm{m}_{1 \mathrm{x}}^{\mathrm{k}} \\
\mathrm{m}_{1 \mathrm{y}}^{\mathrm{k}}-\mathrm{m}_{2 \mathrm{x}}^{\mathrm{k}}=\mathrm{b} & \text { mais } \mathrm{m}_{1 \mathrm{y}}^{\mathrm{k}}+\mathrm{m}_{2 \mathrm{x}}^{\mathrm{k}}=0 \Rightarrow \mathrm{m}_{2 \mathrm{x}}^{\mathrm{k}}=-\mathrm{m}_{1 \mathrm{y}}^{\mathrm{k}} \\
\mathrm{m}_{1 \mathrm{x}}^{\mathrm{k}}-\mathrm{m}_{2 \mathrm{z}}^{\mathrm{k}}=\mathrm{c} & \text { mais } \mathrm{m}_{1 z}^{\mathrm{k}}+\mathrm{m}_{2 z}^{\mathrm{k}}=0 \Rightarrow \mathrm{m}_{2 \mathrm{z}}^{\mathrm{k}}=-\mathrm{m}_{1 z}^{\mathrm{k}}
\end{array}
$$




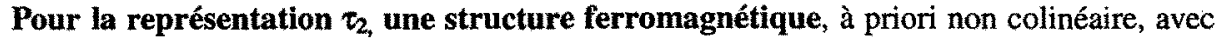
3 paramètres à affiner :

$$
\begin{aligned}
& m_{1 x}^{k}+m_{2 y}^{k}=a \\
& m_{1 y}^{k}+m_{2 x}^{k}=b \\
& m_{1 z}^{k}+m_{2 z}^{k}=c
\end{aligned}
$$

$$
\begin{aligned}
& \text { mais } \mathrm{m}_{1 \mathrm{x}}^{\mathrm{k}}-\mathrm{m}_{2 \mathrm{y}}^{\mathrm{k}}=0 \Rightarrow \mathrm{m}_{2 y}^{\mathrm{k}}=\mathrm{m}_{1 \mathrm{x}}^{\mathrm{k}} \\
& \text { mais } \mathrm{m}_{1 \mathrm{y}}^{\mathrm{k}}-\mathrm{m}_{2 \mathrm{x}}^{\mathrm{k}}=0 \Rightarrow \mathrm{m}_{2 \mathrm{x}}^{\mathrm{k}}=\mathrm{m}_{1 \mathrm{y}}^{\mathrm{k}} \\
& \text { mais } \mathrm{m}_{1 z}^{\mathrm{k}}-\mathrm{m}_{2 z}^{\mathrm{k}}=0 \Rightarrow \mathrm{m}_{2 z}^{\mathrm{k}}=\mathrm{m}_{1 z}^{\mathrm{k}}
\end{aligned}
$$

La comparaison avec les intensités magnétiques montre que la structure est antiferromagnétique comme publié dans Barbara et al. (Solid State Comm. 24, (1977) 481-485) et comme le montre la figure 19. Cependant, au moment de cette détermination, aucune analyse de symétrie n'avait été faite et les auteurs n'avaient affiné qu'un paramètre en supposant de façon tout à fait arbitraire que la structure était colinéaire $\left(\mathrm{m}_{1 \mathrm{x}}^{\mathrm{k}}=\mathrm{m}_{1 \mathrm{y}}^{\mathrm{k}}=\mathrm{m}_{1 \mathrm{z}}^{\mathrm{k}}\right)$. Ceci devait se révéler inexact comme l'ont publié Givord et al. (Physica B, 234-236, (1997),685-686), qui ont montré, par analyse de polarisation que $\mathrm{m}_{1 \mathrm{x}}^{\mathrm{k}}=\mathrm{m}_{\mathrm{iy}}^{\mathrm{k}} \neq \mathrm{m}_{1 z}^{\mathrm{k}}$. Un peu de théorie des groupes au moment de la première détermination de la structure aurait peut-être évité une simplification abusive.
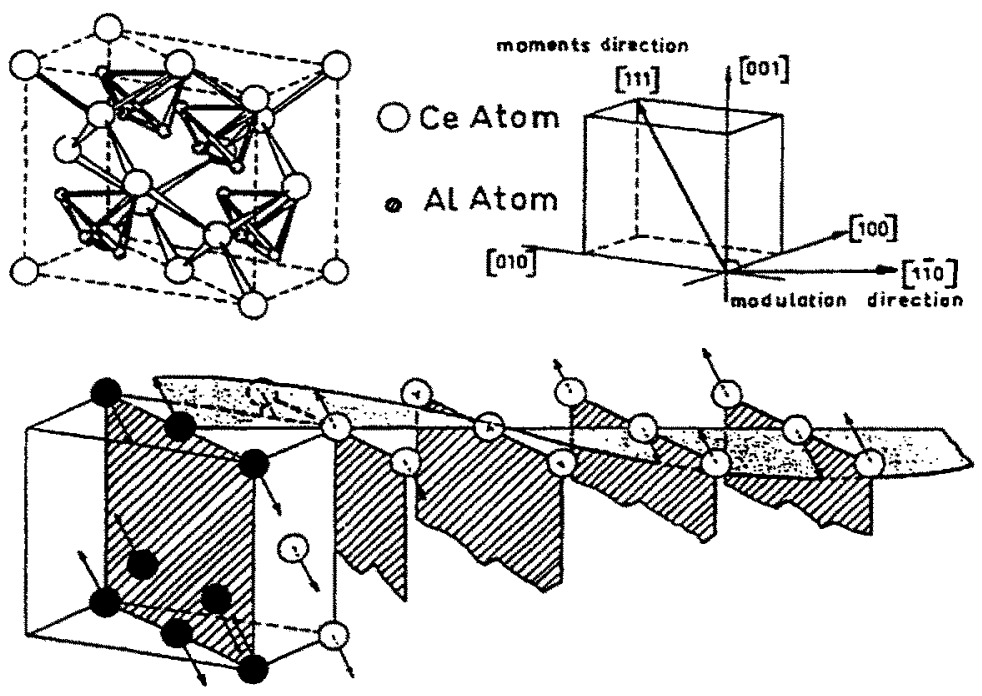

Figure 19 : Structure magnétique de CeAl 2

Pour être complet, il faut ajouter que dans le cas de $\mathrm{CeAl}_{2}$, le passage des composantes de Fourier $\overrightarrow{\mathrm{m}}_{\mathrm{j}}^{\mathrm{k}}$ aux moments magnétiques $\overrightarrow{\mathrm{m}}_{\mathrm{lj}}$ présente une complication (Forgan et al. J. Phys., 2, (1990), 10211-10216) car la structure est multi- $\vec{k}$, c'est à dire que la formule (16) est remplacée par :

$$
\overrightarrow{\mathrm{m}}_{\mathrm{lj}}=\sum_{\overrightarrow{\mathrm{k}}} \overrightarrow{\mathrm{m}}_{\mathrm{j}}^{\overrightarrow{\mathrm{k}}} \mathrm{e}^{-\mathrm{i} \overline{\mathrm{i}}}
$$


où la somme sur $\overrightarrow{\mathrm{k}}$ ne se limite pas à $\overrightarrow{\mathrm{k}}$ et $-\overrightarrow{\mathrm{k}}$, mais à d'autres vecteurs $\overrightarrow{\mathrm{k}}$ équivalents par rapport aux opérateurs de rotation du cristal. L'étude des structures multi- $\vec{k}$ va au delà de ce cours, mais est expliquée dans les articles cités en référence.

\section{REMARQUES}

1) Si dans la décomposition de la représentation vectorielle axiale $\Gamma\left(G_{k}\right)$, la représentation $\tau_{v}$ apparaît plusieurs fois, ou si la représentation $\tau_{v}$ est d'ordre supérieur à un, les solutions possibles sont des combinaisons linéaires des vecteurs de base trouvés.

Il y a une différence théorique entre les deux cas, mais le résultat pratique est le même.

2) Lorsqu'on écrit la représentation vectorielle axiale $\Gamma\left(\mathrm{G}_{\mathrm{k}}\right)$, c'est à dire l'action des éléments $\mathrm{G}_{\mathrm{ki}}$ sur les composantes de départ $\mathrm{m}_{\mathrm{j \alpha \alpha}}^{\mathrm{k}}$, si l'action d'un opérateur envoie la composante d'un atome $\mathrm{j}$ sur un atome $\mathrm{j}^{\prime}$ dans la maille d'à côté (translation $\vec{i}$ ), il ne faut pas oublier le facteur de phase $\mathrm{e}^{-\overrightarrow{\mathrm{k}} \overrightarrow{\mathrm{i}}}$ dans la représentation.

\section{BIBLIOGRAPHIE}

\section{E.F. Bertaut; J. Phys. C1 (1971), 462}

J. Rossat Mignod, Chapter 20: Magnetic Structures, in "Methods of Experimental Physics", edited by K. Skold and D.L. Price, Volume 23, pp 69-157, Acad. Press (1987).

J. Schweizer, Neutron Scattering and Magnetic Structures in "Neutron and Synchrotron Radiation for Condensed Matter Studies" (HERCULES Proceedings), edited by J. Baruchel, J.L. Hodeau, M.S. Lehmann, J.R. Regnard, C. Schlenker, volume II, pp 97-117, Les Editions de Pgysique, Springer-Verlag (1993).

Yu. A. Izyumov, V.E. Naish, R.P. Ozerov, "Neutron Diffraction of Magnetic Materials", Consultant Bureau, New York (1991).

\section{ANNEXE}

Nous allons démontrer dans cette annexe que pour n'importe quelle distribution de moments aux nceuds d'un réseau, on peut écrire :

$$
\overrightarrow{\mathrm{m}}_{1}=\int_{\substack{\text { lere zone } \\ \text { Brillouin }}} \mathrm{d}^{3} \mathrm{k} \overrightarrow{\mathrm{m}}(\overrightarrow{\mathrm{k}}) \mathrm{e}^{-\mathrm{i} \overrightarrow{\mathrm{k}} \mathrm{i}}
$$

où $\overrightarrow{\mathrm{m}}(\overrightarrow{\mathrm{k}})$ est une distribution vectorielle continue et périodique dans le réseau réciproque.

Il est bien connu que si on a dans l'espace direct une densité périodique avec la périodicité du réseau $\rho(\overrightarrow{\mathrm{r}}+\overrightarrow{\mathrm{l}})=\rho(\overrightarrow{\mathrm{r}})$, la transformée de Fourier de cette densité $\rho(\overrightarrow{\mathrm{r}})$ est un ensemble de pics $\delta$ aux noeuds $\vec{\tau}$ du réseau réciproque et que la valeur de ces pies est donnée par la transformée de Fourier: 


$$
\mathrm{F}(\vec{\tau})=\int_{\text {maille }} \rho(\overrightarrow{\mathrm{r}}) \mathrm{e}^{i \vec{\tau} \mathrm{r}} \mathrm{d}^{3} \mathrm{r}
$$

Inversement, si on part dans le réseau réciproque d'une distribution d'amplitudes $F(\vec{k})$ sous forme de pics $\delta$ aux nœuds $\vec{\tau}$ du réseau réciproque, la transformée de Fourier inverse :

$$
\rho(\overrightarrow{\mathbf{r}})=\sum_{\overrightarrow{\mathrm{r}}} \mathrm{F}(\vec{\tau}) \mathrm{e}^{-\overrightarrow{\mathrm{r}} \mathrm{r}}
$$

donnera comme fonction $\rho(\overrightarrow{\mathrm{r}})$ dans le réseau direct une fonction continue et périodique, telle que :

$$
\rho(\overrightarrow{\mathrm{r}}+\overrightarrow{\mathrm{l}})=\rho(\overrightarrow{\mathrm{r}})
$$

Sachant que le réseau réciproque du réseau réciproque est le réseau direct, si on part maintenant d'une distribution quelconque d'amplitudes $m(\overrightarrow{1})$ aux nœuds du réseau réel, sa transformation de Fourier :

$$
\rho(\vec{k})=\sum_{\vec{i}} m(\vec{l}) e^{i \vec{k}]}
$$

donnera pour $\rho(\overrightarrow{\mathrm{k}})$ une fonction continue et périodique dans l'espace réciproque :

$$
\rho(\vec{k}+\vec{\tau})=\rho(\vec{k})
$$

et on peut revenir à la distribution $\mathrm{m}(\overrightarrow{\mathrm{l}})$ par la transformée de Fourier inverse :

$$
m(\vec{l})=\int_{\substack{\text { lere zane } \\ \text { Brillouin }}} \rho(\vec{k}) \mathrm{e}^{-i \vec{k} \mathbf{l}} \mathrm{d}^{3} \mathrm{k}
$$

Si maintenant on part d'une distribution vectorielle $\vec{m}(\vec{l})$ aux nœuds du réseau réel, il suffit d'appliquer la formule (61) aux trois composantes de ces vecteurs pour aboutir à une transformée de Fourier:

$$
\vec{\rho}(\vec{k})=\sum_{\vec{i}} \vec{m}(\vec{l}) e^{i \vec{k} \vec{i}}
$$

qui soit une fonction vectorielle, continue et périodique dans l'espace réciproque:

$$
\vec{\rho}(\vec{k}+\vec{\tau})=\vec{\rho}(\vec{k})
$$

et un retour à la distribution vectorielle $\overrightarrow{\mathrm{m}}(\overrightarrow{\mathrm{l}})$ de l'espace réel en appliquant la transformée de Fourier inverse (63) aux trois composantes de la distribution $\vec{\rho}(\overrightarrow{\mathrm{k}})$ :

$$
\overrightarrow{\mathrm{m}}(\overrightarrow{\mathrm{l}})=\int_{\substack{\text { lere zose } \\ \text { Brillouin }}} \vec{\rho}(\overrightarrow{\mathrm{k}}) \mathrm{e}^{-i \overrightarrow{\mathrm{k}} \overrightarrow{3}} \mathrm{~d}^{3} \mathrm{k}
$$

qui est équivalente à la formule (3). 